\title{
Single-cell RNA sequencing identifies inflammatory tissue T cells in eosinophilic esophagitis
}

\author{
Ting Wen, ${ }^{1}$ Bruce J. Aronow, ${ }^{2}$ Yrina Rochman, ${ }^{1}$ Mark Rochman, ${ }^{1}$ Kiran KC, ${ }^{1}$ Phil J. Dexheimer, ${ }^{2}$ Philip Putnam, ${ }^{3}$ Vincent Mukkada, ${ }^{3}$ \\ Heather Foote, ${ }^{1}$ Kira Rehn, ${ }^{1}$ Sam Darko, ${ }^{4}$ Daniel Douek, ${ }^{4}$ and Marc E. Rothenberg ${ }^{1}$ \\ 'Division of Allergy and Immunology, 2Division of Biomedical Informatics, and ${ }^{3}$ Division of Castroenterology, Hepatology and Nutrition, Department of Pediatrics, Cincinnati Children's Hospital Medical \\ Center, University of Cincinnati, Cincinnati, Ohio, USA. ${ }^{4}$ Human Immunology Section, Vaccine Research Center, National Institute of Allergy and Infectious Diseases, National Institutes of Health, \\ Bethesda, Maryland, USA.
}

\begin{abstract}
T cell heterogeneity is highly relevant to allergic disorders. We resolved the heterogeneity of human tissue $\mathrm{CD3^{+ }} T$ cells during allergic inflammation, focusing on a tissue-specific allergic disease, eosinophilic esophagitis (EoE). We investigated 1088 single $\mathrm{T}$ cells derived from patients with a spectrum of disease activity. Eight disparate tissue $\mathrm{T}$ cell subtypes (designated T1-T8) were identified, with T7 and T8 enriched in the diseased tissue. The phenotypes of T7 and T8 resemble putative Treg (FOXP3 ${ }^{+}$) and effector Th2-like (CATA3 ${ }^{+}$) cells, respectively. Prodigious levels of IL-5 and IL-13 were confined to HPCDS CRTH2+IL-17RB+FFAR3+CD4+ T8 effector Th2 cells. EoE severity closely paralleled a lipid/fatty acid-induced activation node highlighted by the expression of the short-chain fatty acid receptor FFAR3. Ligands for FFAR3 induced Th2 cytokine production from human and murine T cells, including in an in vivo allergy model. Therefore, we elucidated the defining characteristics of tissue-residing $\mathrm{CD}^{+} \mathrm{T}$ cells in EoE, a specific enrichment of $\mathrm{CD4}{ }^{+}$Treg and effector Th2 cells, confinement of type 2 cytokine production to the CD4+ effector population, a highly likely role for FFAR3 in amplifying local Th2 responses in EoE, and a resource to further dissect tissue lymphocytes and allergic responses.
\end{abstract}

\section{Introduction}

Substantial heterogeneity of $\mathrm{CD}^{+} \mathrm{T}$ cells exist, starting grossly with $\mathrm{CD}^{+}$and $\mathrm{CD} 8^{+}$cells, which are involved in helper and cytotoxic responses, respectively. $\mathrm{CD}^{+} \mathrm{T}$ helper (Th) cells, as the orchestrators of adaptive immunity, are divided into cells that secrete discrete families of cytokines such as Th1, Th2, and Th17 cells (1). Imbalanced levels or activity of these cells results in substantial consequences regarding susceptibility to infections, cancer, allergy, and autoinflammatory diseases; thus, there is considerable regulatory control of $\mathrm{T}$ cell responses, largely mediated by Tregs. Most studies focused on characterizing these cells in humans have been conducted on circulating cells, despite that most $\mathrm{T}$ cell effector function occurs in the tissue.

Herein, we applied single-cell RNA sequencing (scRNA-seq) to profile 1088 human $\mathrm{T}$ cells residing in the tissues of subjects with healthy and allergic states to reveal the diversity of transcriptional phenotypes that can be adopted by tissue $\mathrm{T}$ cells. We

\section{Related Commentary: p. 1830}

Conflict of interest: MER is a consultant for Pulm One, Spoon Guru, ClostrBio, Celgene, Shire, Astra Zeneca, Glaxosmithkline, Allakos, Adare, Regeneron, and Novartis; has an equity interest in the first 3 companies listed and in Immune Pharmaceuticals; and receives royalties from reslizumab (Teva Pharmaceuticals) and UpToDate. MER and TW are the inventors of patents owned by Cincinnati Children's Hospital (patent nos. 9,928,34B2, PCT/US12/43640, and 62/748,754).

Copyright: @ 2019, American Society for Clinical Investigation.

Submitted: October 31, 2018; Accepted: February 21, 2019; Published: April 8, 2019.

Reference information: J Clin Invest. 2019;129(5):2014-2028.

https://doi.org/10.1172/JCl125917. focused on analyzing a model, tissue-specific, allergic disease termed eosinophilic esophagitis (EoE), as this is a food antigen-driven disease involving adaptive T cell memory (2). EoE is dependent on cytokines likely derived from T cells (e.g., IL-13), as evidenced by recent successful clinical trials with anti-IL-13 and anti-IL-4R $\alpha$ antibody therapy (3-5). Importantly, human tissue samples enriched in $\mathrm{T}$ cells were readily available via endoscopy, providing an opportunity to probe human tissue $\mathrm{T}$ cells at the single-cell level (Figure 1A). To fill the gaps in understanding the functionality of tissue lymphocytes, we concentrated on several driving queries, including (a) what is the heterogeneity of tissue $\mathrm{CD}^{+} \mathrm{T}$ cells, (b) how distinct are tissue $\mathrm{CD}^{+} \mathrm{T}$ cells from their circulating counterparts, (c) do any EoE-specific T cell subpopulations exist and how do they contribute to pathogenesis, and (d) are there any natural agents from food source/microbiota that could regulate the pathogenesis of disease-specific tissue T cells?

\section{Results}

Building the human experimental single-cell platforms. We first probed single-cell suspensions from human endoscopic biopsies by FACS, focusing on CD45/CD3 double-positive cells and comparing them to cells obtained from autologous blood (Figure 1, A and B). iNKT cells (defined by invariant TCR Va24-Ja18) and B cells (defined by CD19) did not represent a substantial population of cells from this mucosal tissue (Supplemental Figure 1, A and B; supplemental material available online with this article; https://doi.org/10.1172/ JCI125917DS1). T cells ( $\alpha \beta)$ markedly dominated over $\delta \gamma \mathrm{T}$ cells in cellularity, at both the protein (Supplemental Figure 1C) and mRNA (Supplemental Figure 1D) levels. The total number of $\mathrm{T}$ 


\section{A}

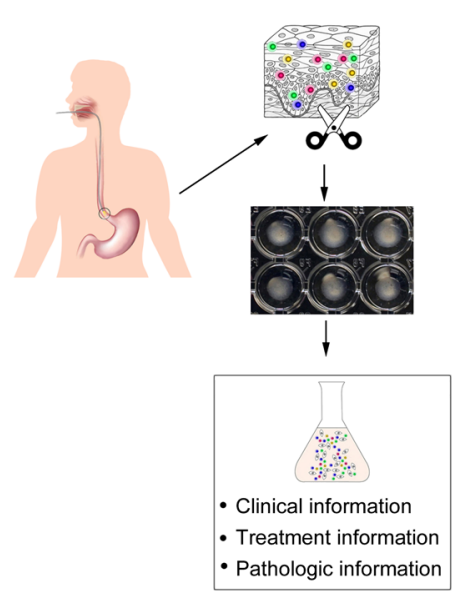

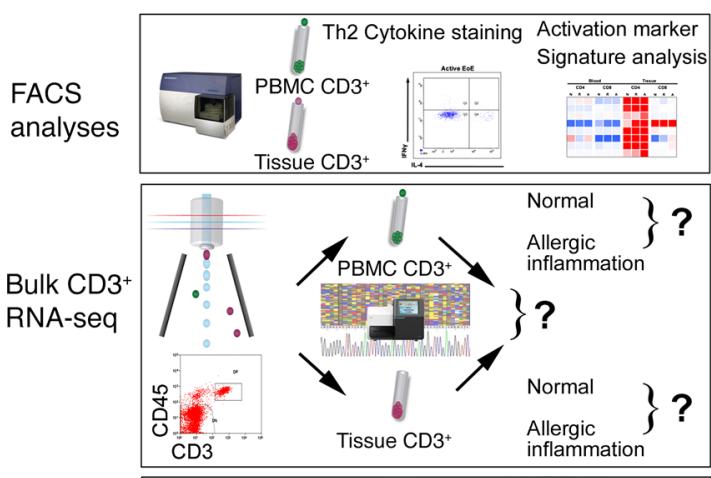

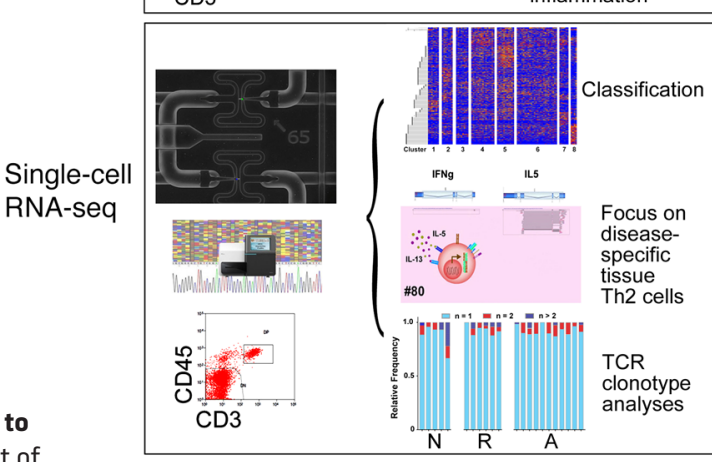

Figure 1. The schematic overall strateg study tissue lymphocytes. (A) Flow chart of the overall single-cell platform used to study human tissue lymphocytes, which consists of 3 basic modules: flow cytometry (FACS), bulk $\mathrm{CD}^{+}$RNA-seq, and C1 Fluidigm-based single-cell RNA-seq. (B) $\mathrm{CD}^{+}$lymphocytes isolated from esophageal biopsy tissue and autologous blood were analyzed by FACS and substratified into $\mathrm{CD} 4^{+}$and $\mathrm{CD} 8^{+}$populations.

\section{B}

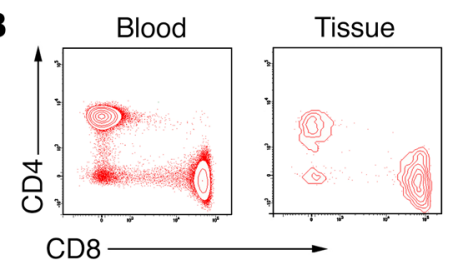

itive for the transcription factor FOXP3 (Figure 2E), consistent with being tissue residential Tregs. Similar to circulating Tregs, these tissue CD $25^{+}$cells exhibited a $\mathrm{CD} 127^{\mathrm{lo}}, \mathrm{CD} 45 \mathrm{RO}^{+}$, and CTLA4 ${ }^{+}$phenotype, suggesting that they resemble memory effector Tregs (Figure 2E).

Bulk $\mathrm{CD}^{+}$RNA-seq identifies specific gene cohorts related to tissue and allergic inflammation. Regardless of disease status, there were 331 unique genes significantly differentially expressed between autologous blood and tissue $\mathrm{CD}^{+} \mathrm{T}$ cells using stringent criteria (FDR-adjusted $P$ $<0.05$, fold change $>10$, paired moderated 2-tailed $t$ test) (Figure 3A; full gene list in Supplemental Table 1 and full pathway analysis in Supplemental Table 2). We found functional nodes involved in leukocyte migration, defensive response to gastrointestinal stimuli, and regulated exocytosis (Supplemental Figure 2A). Likewise, the FDR-adjusted $P$ valueprioritized approach indicated functional nodes enriched in gastrointestinal anatomical structure development, multicellular communication, and immune response to stimuli (Supplemental Figure 2B). A cohort of 150 genes that were different between normal and active disease tissue lymphocytes was identi-

cells enumerated from a single biopsy positively correlated with disease severity for both CD4 and CD8 compartments and was significantly different between control individuals (referred to as normal) and subjects with allergy in both $\mathrm{CD} 4^{+}$(3.3-fold increase, $P$ $<0.05$, 2-tailed $t$ test) and CD8 ${ }^{+}$(5.1-fold increase, $P<0.01$, 2-tailed $t$ test) populations (Figure 2A), reflecting an overall lymphocytosis in the allergic mucosa ( $\mathrm{CD}^{+}$enumeration per biopsy: $542 \pm 288$ for normal, $778 \pm 830$ for inflammation remission, and $2189 \pm 1661$ for active inflammation, mean $\pm \mathrm{SD}$ ). A significant $\mathrm{CD}^{+} \mathrm{T}$ cell dominance was found in the tissue compared with the typical $\mathrm{CD} 4^{+} \mathrm{T}$ cell dominance in the circulation (Figure 2B).

$\mathrm{CD} 25 / \mathrm{IL}-2 \mathrm{R} \alpha^{+}$events were found to be overrepresented in tissue samples with active EoE compared with samples with inflammation in remission or lack of EoE presence/history (Figure 2C, $P<0.01$, 2-way ANOVA/Bonferroni posttesting). Although CD25 expression was significantly augmented in tissue CD4 ${ }^{+}$cells compared with their blood counterparts (Figure 2C, $P<0.001$, 2-way ANOVA), no statistical difference of CD25 expression was found within the circulating $\mathrm{CD}^{+} \mathrm{T}$ cell compartment in the context of the inflammation activity (2-way ANOVA/Bonferroni posttesting). As a control, CD25 expression was barely observed in $\mathrm{CD}^{+} \mathrm{T}$ cells (Figure 2C). A robust correlation between $\mathrm{CD} 25^{+} \mathrm{CD} 4^{+}$events and eosinophil levels was seen in the tissue but not the circulation (Figure 2D, $P<0.001$, Spearman $r=0.65$ for tissue correlation). CD25, the IL-2 receptor $\alpha$ chain, is a component of activated T cells as well as a feature of Tregs. The CD $25^{+}$cells identified were pos- fied, with 147 of the genes being upregulated in allergic compared with control tissue T cells (Figure 3B, and Supplemental Table 3 , FDR-adjusted $P<0.05$, fold change $>10$, moderated 2 -tailed $t$ test). Functional prediction analysis of these 150 disease-associated genes indicated enrichment of pathways involved in granulocyte degranulation, activation, migration, and immune exocytosis (Supplemental Figure 3A and Supplemental Table 3). Representative Th2-related genes are shown across all conditions (Figure 3C). Because the lymphocyte transcript signature did not completely normalize following remission (Figure 3B and Supplemental Figure 2C), remission tissue lymphocytes were genetically compared with active inflammation and normal tissue lymphocytes for genes potentially accounting for EoE relapse. With genome-wide Mann-Whitney tests, a cohort of 217 genes was found to be dysregulated between remission and active inflammatory disease and a cohort of 333 genes was dysregulated between remission and normal lymphocytes (Supplemental Figure 2C, FDR-adjusted $P<0.05$, fold change $>2$, and Supplemental Table $4, \mathrm{~A}$ and B).

The flow cytometry finding of the overabundance of $\mathrm{CD} 25^{+}$ T cells in allergic tissue (Figure 2, C-E) was substantiated by bulk RNA-seq of $\mathrm{CD}^{+} \mathrm{T}$ cells. The major Th2 cytokines, namely IL-4, IL-5, and IL-13, were robustly expressed by tissue lymphocytes present in EoE, with the gene of the eosinophil-directed cytokine IL- 5 being the most dysregulated (Figure 3C). IL 5 demonstrated a 1500-fold increase in cells from active EoE compared with control tissue. Of note, the gene of the classical Th2 marker IL-17RB 

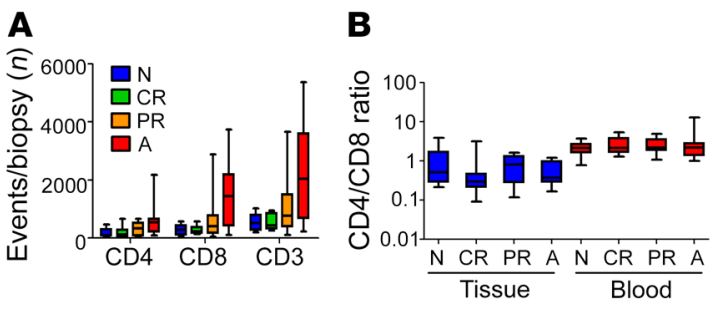

\section{c}
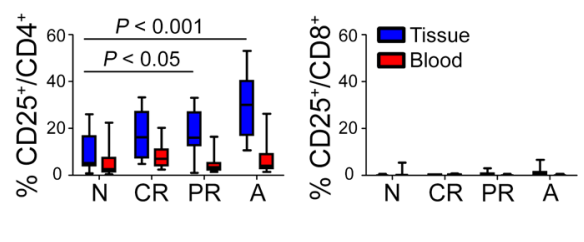

D

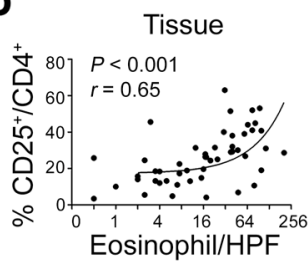

E Blood

Tissue

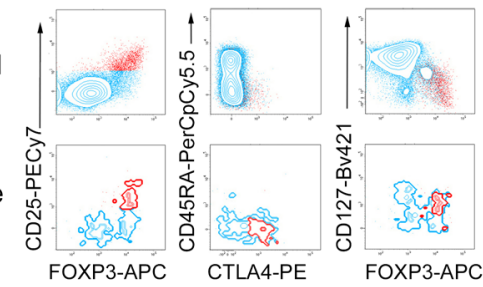

Figure 2. FACS analysis of $\mathrm{CD3}^{+} \mathbf{T}$ cells in blood and tissue. (A) The number of $\mathrm{CD4^{+ }}, \mathrm{CD} 8^{+}$, and pan $\mathrm{CD}^{+} \mathrm{T}$ cells isolated from a single biopsy were enumerated from 4 different cohorts: normal ( $N, n=9)$, complete remission (CR, $n=6$ ), partial remission (PR, $n=12$ ), and active inflammation (,$n=13$ ). Two-way ANOVA, EoE factor: $P<0.001$; CD4/CD8 factor: $P<0.001$; interaction, nonsignificant. (B) With the same 4 disease cohorts, the CD4/CD8 ratio was quantified from autologous blood and tissue samples. Two-way ANOVA, EoE factor nonsignificant; tissue/blood factor: $P<0.001$; interaction, nonsignificant. (C) The percentages of $C D 25^{+}$events were quantified by normalizing to gated $\mathrm{CD}^{+}$or $\mathrm{CD} 8^{+}$events. CD25 $/ \mathrm{CD} 4^{+}$2-way ANOVA, EoE factor: $P<0.001$; tissue/blood factor: $P<0.001$; interaction, $P<0.01$. Bonferroni test: $P<0.05$, normal controls (N) versus $P R ; P<0.001$, $N$ versus $A$. (D) The percentage of $\mathrm{CD} 25^{+} \mathrm{CD} 4{ }^{+}$cells was correlated with tissue eosinophilia, but not autologous blood eosinophilia, with the $P$ value and Spearman $r$ shown. (E) Tissue and autologous blood $\mathrm{CD3}^{+} \mathrm{CD4}^{+}$events (blue) plotted in the context of multiple proteins of interest, with CD25 events labelled in red. Two-way ANOVA was used for multi-comparisons in C, D and E. Box and whisker plots are shown indicating mean and minimum/maximum values.

(the IL-25 receptor) was upregulated in EoE tissue lymphocytes, with the gene of the recently reported pathogenic effector Th2 cell marker HPGDS (6) being upregulated in a similar fashion (Figure 3C). The top 10 dysregulated genes in tissue lymphocytes were graphed for fold-change and $P$ values and selected for a pattern analysis across all conditions, resulting in 3 distinct expression patterns (Figure 3D). We performed 2-way ANOVA in EoE and control blood and tissue to screen for differentially expressed integrin and chemokine receptor genes in the contexts of blood versus tissue location and EoE status (Supplemental Figure 3B). A large proportion of both categories (14/23 for chemokine receptor and $10 / 27$ for integrins) passed the FDR filter $(P<0.05)$, showing tissue-blood differences in a bidirectional fashion. We did not find any disease-specific change or any interactions, indicating that these processes were homeostatic events. ITGAE (CD103), a mucosal $\mathrm{T}$ lymphocyte differential marker and migration adhesion cue in humans and mice $(7,8)$, served as a positive control. In the context of tissue comparison, integrin $\mathrm{A} 1, \mathrm{AV}, \mathrm{B} 4$, and $\mathrm{B} 8$ were the most upregulated integrins in tissue $\mathrm{CD}^{+}$cells. Likewise, the Th2-prone memory/effector chemokine receptors (9-11) CXCR4, CCR5, CCR8 - were among the most upregulated receptors (Supplemental Figure 3B), suggesting that the tissue compartment may be Th2-promoting to a certain extent regardless of EoE status. CCR3 and CCR7 were downregulated in tissue $\mathrm{CD}^{+}$cells (Supplemental Figure 3B).

ScRNA-seq identifies 8 principal tissue T cell subtypes. We utilized the C1 Fluidigm system to cumulatively capture 1088 CD3/ CD45-sorted live T cells from 17 subjects with active EoE (11 with active inflammation and 6 with resolved inflammation) and 5 nor- mal control individuals (clinical and demographical parameters are summarized in Supplemental Table 5). The work flow is schematically summarized in Supplemental Figure 4A. Live cells were selected by FACS sorting of $\mathrm{CD}^{+} \mathrm{CD}^{4} 5^{+}$and each single $\mathrm{T}$ cell was examined microscopically after it was loaded onto the C1 Fluidigm chip. Each cell was targeted for 2 million pair-ended reads. Alignment to hg19 human genome annotation (GRCh37 assembly) yielded 45,987 unique aligned entities. During quality control measures, only one duplet was found, and it was excluded from the analysis, resulting in a total pool of $1088 \mathrm{CD}^{+}$tissue lymphocytes. By the random C1 Fluidigm capture, we obtained 584, 265, and 239 tissue lymphocytes from subjects with active EoE, inactive EoE, and control subjects, respectively, which is consistent with the increased $\mathrm{T}$ cell cellularity differences observed by FACS (Figure 2A). Next, an unsupervised approach to define tissue $\mathrm{T}$ cell subtypes was performed by principal components analysis (PCA) to screen for those genes whose expression most contributed to the observed variations among different subgroups. Briefly, a summation of the top contributing genes in each unique cluster/component was assembled into a collective cohort of 1114 genes contributing to the interclass variations in tissue lymphocytes present in EoE (Supplemental Figure 4A, Supplemental Table 6). These genes formed the basis for identifying 8 unique $\mathrm{T}$ cell populations, designated T1-T8 (Figure 4, A-E). A heatmap of the select cohort of genes that differentiates T1-T8 is shown (Supplemental Figure 4B). No global changes in transcriptional levels were a function of T1-T8 classification, EoE disease activity, or disease severity (as assessed by tissue eosinophilia) (Supplemental Figure 5A) despite the vast heterogeneity and local $\mathrm{T}$ cluster-specific activation described later. The composition of CD4 and CD8 (including both moieties of the CD8 dimer) was analyzed by 3D reconstruction and showed dichotomy between CD4 and CD8A/B (Supplemental Figure 5B), with the CD4 component being highly enriched in the active inflammatory disease group. Though most CD8 cells were $\mathrm{CD} 8 \alpha \beta, \mathrm{CD} 8 \alpha \alpha$ (likely marking natural intraepithelial lymphocytes) was more frequent than $C D 8 \beta \beta$. There was no detectable difference in disease activity as a function of $\mathrm{CD} 8 \alpha / \mathrm{CD} 8 \beta$ composition, except for the disease-specific enrichment of the CD4 component (Supplemental Figure 5B). We observed a scarce proportion of CD4/CD8 double-positive events particularly in active inflammatory disease, consistent with a surface phenotype of activated memory CD4 ${ }^{+}$ $\mathrm{T}$ cells (12). Additionally, CD4-CD8A- double-negative $\mathrm{CD}^{+}$cells 
A
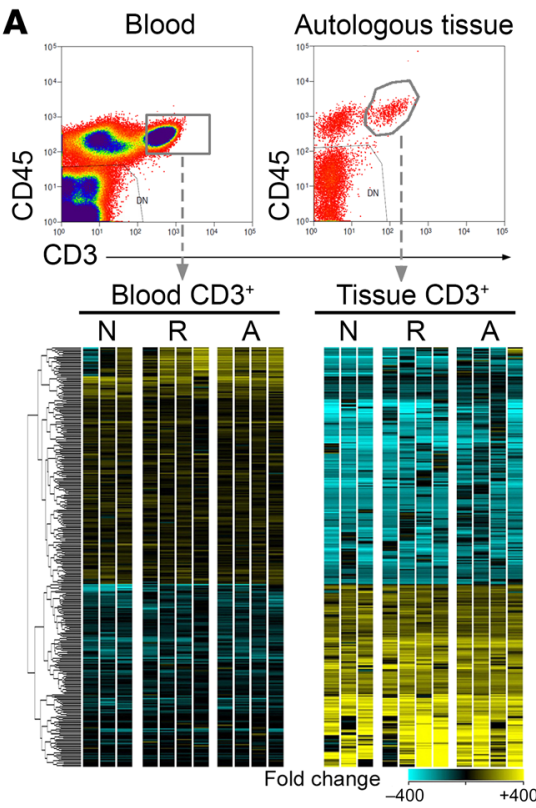

B

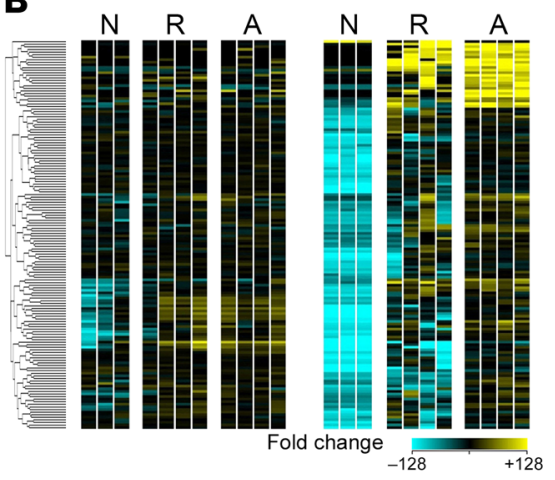

C
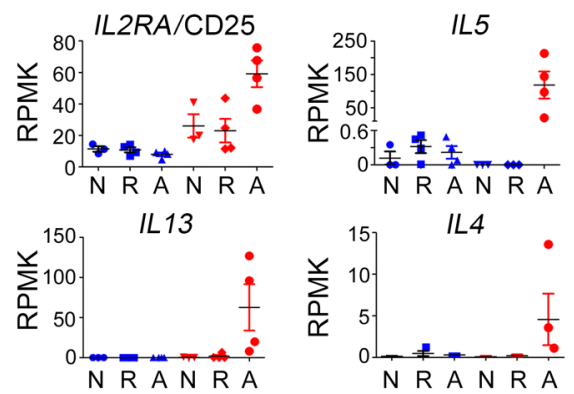

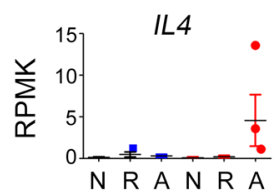

HPGDS
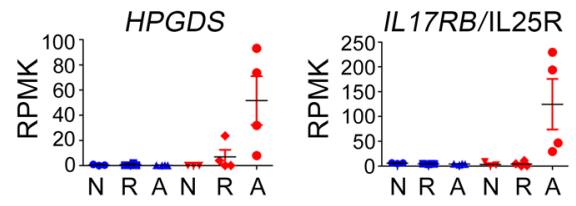

D

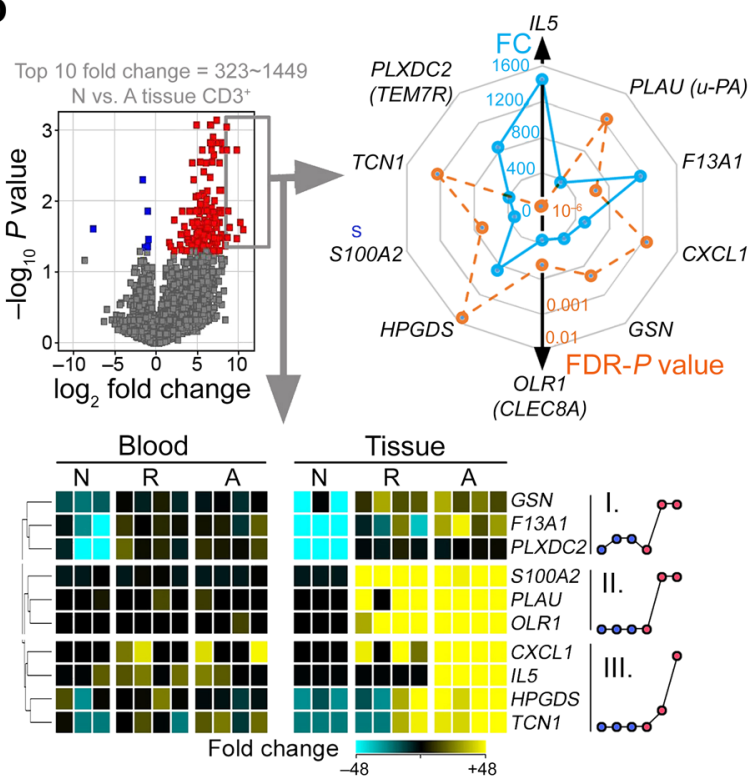

Figure 3. Bulk CD3 ${ }^{+}$RNA-seq analysis of tissue lymphocytes. (A) $\mathrm{CD}^{+} \mathrm{T}$ cells were sorted from autologous blood and tissue from control (normal [N], $n=3$ ) individuals and patients with EoE with active (active $[A], n=4$ ) or inactive (complete remission $[R], n=4$ ) EoE for bulk RNA-seq analysis. The bidirectional expression profile difference between blood and tissue T cells are displayed in the heat diagram, with each column representing a patient sample. A set of 331 genes reflected tissue-specific functions (paired moderated $t$ test; FDR-adjusted $P<0.05$, fold change $>10$ ). (B) Gene expression (150 genes) tracking with disease activity, 147 of which were significantly upregulated and 3 of which were downregulated in active EoE tissue lymphocytes compared with healthy controls (moderated $t$ test, FDR-adjusted $P<0.05$, fold change $>10$ ). (C) Among the 147 genes in B, the expression trends of 6 Th2-related genes across tissue and disease states are shown (blue: autologous blood CD3+; red: tissue CD3+). (D) The top 10 genes (fold change [FC] = 323-1449, NL tissue $\mathrm{CD}^{+}$vs. EoE tissue $\mathrm{CD}^{+}$) from the volcano plot are displayed in a heat diagram. The expression pattern among the 6-comparison group is summarized in the adjacent dot plots showing 3 distinct trends labelled by roman numerals I-III. The fold change (FC, blue) and the FDR-adjusted $P$ value (FDR- $P$, orange) were exhibited in a radar map in the context of the 10 top genes.

were detectable independent of disease activity by FACS (Figure 1B) and scRNA-seq (Supplemental Figure 11). A consistent portion of all $\mathrm{T}$ cell clusters (T1-T8) exhibited a classical tissue residential memory (TRM) surface phenotype of $\mathrm{CD} 69^{+} \mathrm{CD} 103^{+}$(Supplemental Figure 6). Collectively, we identified T1-T6 cells largely as $\mathrm{CD} 8^{+}$cells and $\mathrm{T} 7-\mathrm{T} 8$ cells largely as $\mathrm{CD} 4^{+}$cells.

Identification of 2 overrepresented $T$ cell subpopulations. We reduced the dimensionality of the genetic variation of the T1T8 clusters to a 2D plot using $t$-distributed Stochastic Neighbor Embedding (t-SNE) (Figure 4A and Supplemental Figure 4A) (13). Most notably, clusters T7 and T8 segregated distinctively from T1T6 (Figure 4A and Supplemental Figure 6A). T8 was dramatically overrepresented in EoE tissue, followed by T7 (Figure 4A). The levels of $\mathrm{T} 7$ and $\mathrm{T} 8$ as a function of total $\mathrm{CD} 3^{+} \mathrm{T}$ cells were $11 \%$ and $6.4 \%$ in subjects with active EoE, $3 \%$ and $0.4 \%$ in subjects with EoE remission, and $4.6 \%$ and $0 \%$ in control individuals, respectively. The composition of T1-T8 as a function of the $3 \mathrm{EoE}$ disease states is presented in Figure 4B. A heat diagram that depicts the single-cell presence for each of the 8 clusters broken down as a function of inflammatory disease activity is shown in Supplemental Figure 4C. Importantly, the overall cellularity increase in allergic inflammation was highlighted by $\mathrm{T} 7$ and $\mathrm{T} 8$, which had a pronounced overrepresentation in patients with active EoE (Figure 4, A and B and Supplemental Figure 4C). 
A

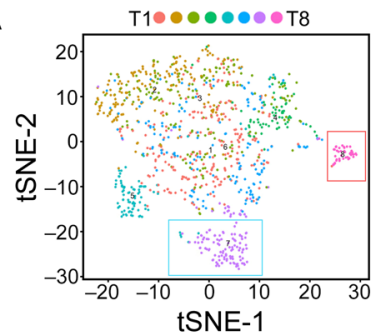

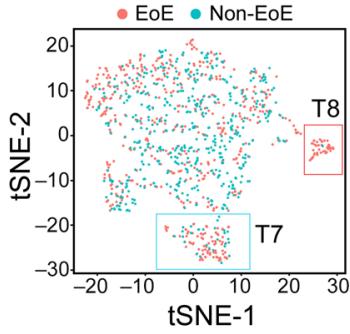

tSNE-1

B

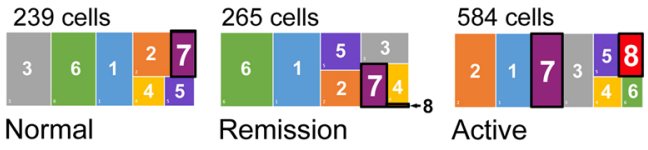

c

1

2

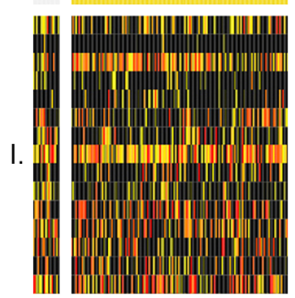

II.
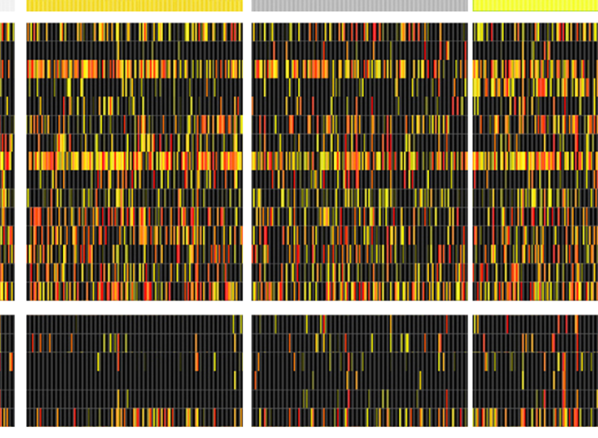

3

$\begin{array}{llll}5 & 6 & 7 & 8\end{array}$
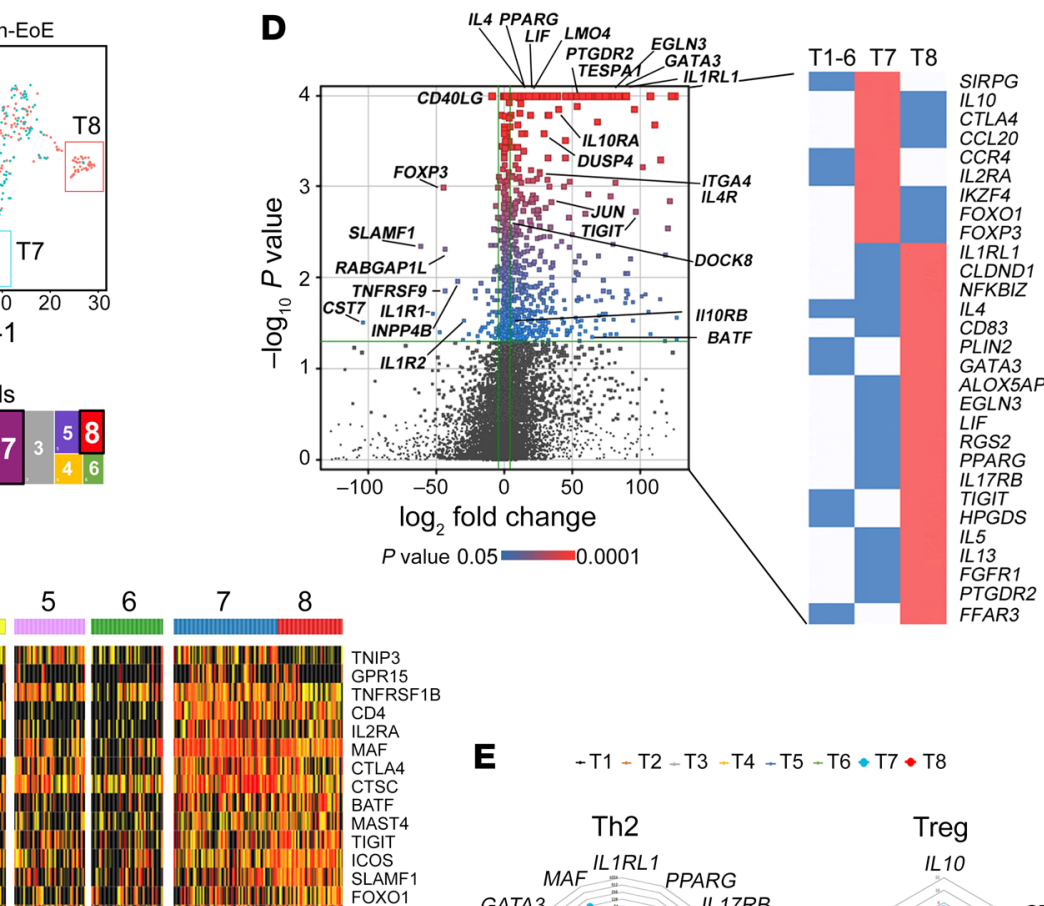

E $\quad-\mathrm{T} 1-\mathrm{T} 2-\mathrm{T} 3-\mathrm{T} 4-\mathrm{T} 5-\mathrm{T} 6 * \mathrm{~T} 7 \bullet \mathrm{T} 8$
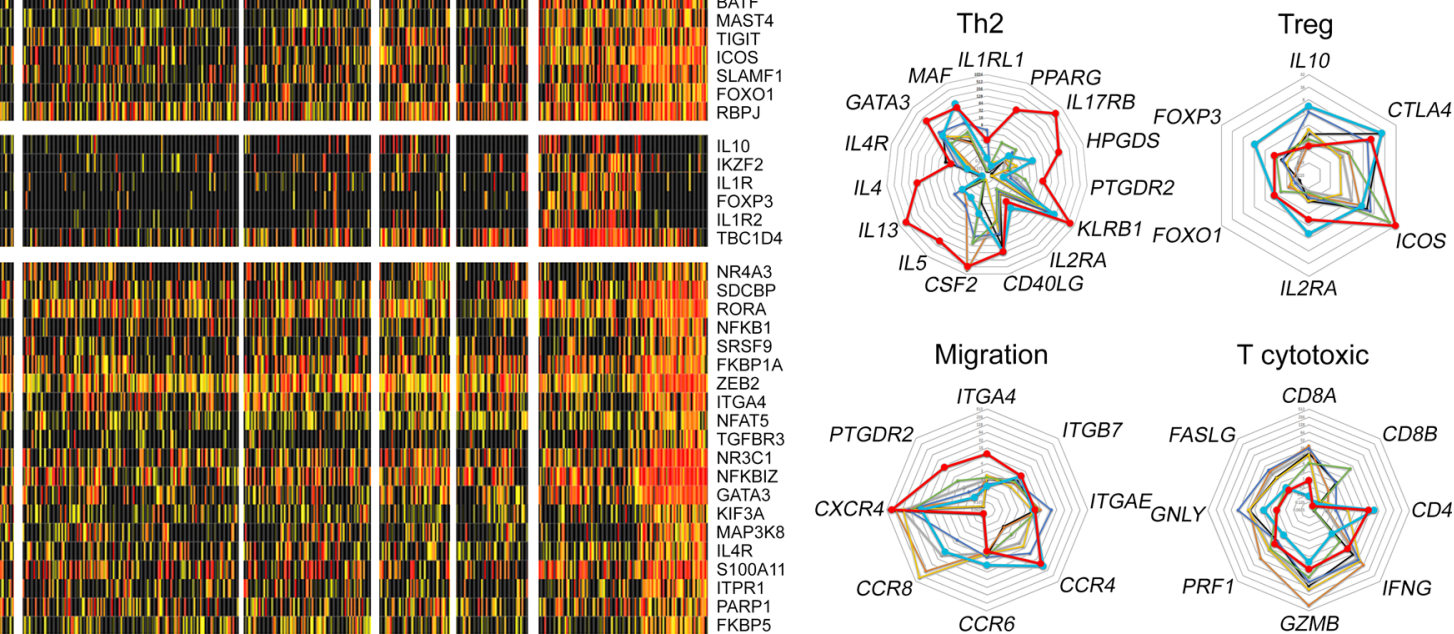

RBPJ

III.
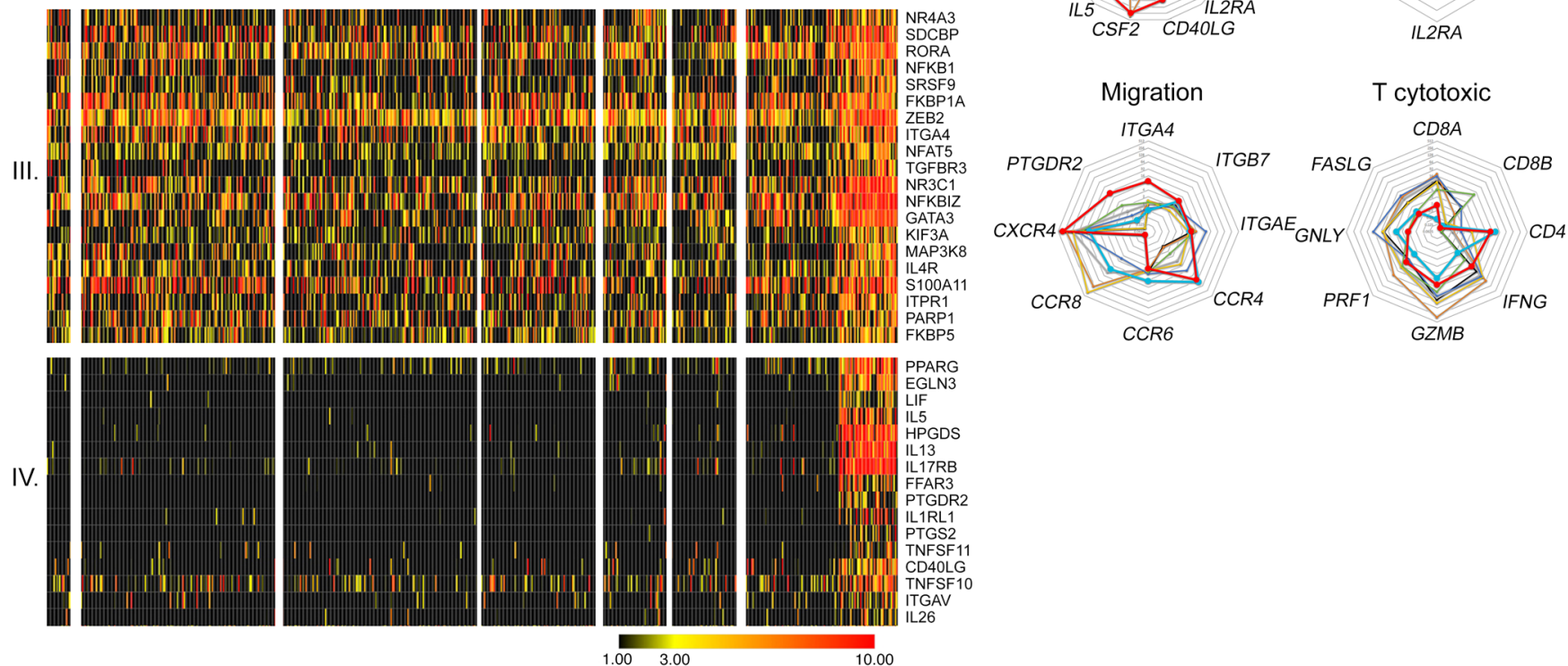

Figure 4. scRNA-seq identification of 8 lymphocyte subpopulations. (A) Left panel: with t-SNE dimensionality reduction, the T1-T8 clusters were 2D plotted with each tissue T cell subclass color coded as indicated. Right panel: a 2D plot was color coded in the context of patients with and without EoE (non-EoE group includes NL and EoE remission). (B) With the large rectangle representing all single T cells isolated from NL, EoE remission, and active EoE subjects, the cellularity presence of T1-T8 subclasses was proportionally graphed within the disease status bin that was set to the equal area as $100 \%$. (C) To emphasize the expression profiles of T7 and T8, a heatmap was selectively generated focusing on the T7-specific Treg genes and the T8-specific Th2-functionality genes, as well as those genes common to both clusters. Four patterns were identified on the basis of differential expression. Patterns I, II, III, and IV were enriched with distinct T clusters-I with T7+T8, II with T7 only, III with ubiquitous T clusters but T8 enriched, and IV with T8 only. (D) A volcano plot comparing top differentially expressed genes between $T 7$ and T8, with key significant genes labeled. Colorized entities represent passing the filter of FDR-adjusted $P<0.05$ and fold change $>16$ with red-blue gradient on FDR-adjusted $P$ values. (E) A series of radar plots based on relevant functional gene sets was generated on logarithm scale to depict the expression characteristics of T1-T8 restricted to Th2 cell markers and cytokines, Treg properties, migration molecule expression related to chemotaxis and adhesion, and T cytotoxic cells properties, respectively. T7 (blue line) and T8 (red line) are shown in bold to emphasize the 2 disease-associated clusters. 
Defining the immunological identity of $\mathrm{T} 7$ and T8. With the T1T8 defined by PCA (Figure 4A and Supplemental Table 6), we next contrasted the signatures of the disease-specific $\mathrm{T} 7$ and $\mathrm{T} 8$ alongside T1-T6 (T7+T8 vs. T1-T6, Figure 4C), further substratifying the genes into 4 cohorts with distinct patterns. Patterns I, II, III, and IV were enriched with distinct T clusters: I with T7+T8, II with T7 only, III with ubiquitous T clusters but T8 enriched, and IV with T8 only. T7 and T8 were the only 2 CD4-enriched clusters, with T7 having a Treg phenotype $\left(\mathrm{CD}^{+} \mathrm{MAF}^{+} \mathrm{FOXP}^{+} \mathrm{CTLA}^{+} \mathrm{IL}^{-} 10^{+}\right)$ and $\mathrm{T} 8$ expressing uniquely high levels of Th2 cytokines, GATA3, and Th2 signature markers (Figure 4, C-E). All T clusters were found to be highly positive for KLRB1 (CD161), with the highest levels expressed in T7 and T8 (Figure 4E), which is consistent with a recent finding that allergen-specific $\mathrm{T}$ effector cells are marked by Th2 cytokines and CD161 (14).

Radar scanning identified closeness of genes of major cytotoxic $\mathrm{T}$ cell components (Figure $4 \mathrm{E}$ ), such as perforin, granzyme, and interferon- $\gamma$, and cytotoxic T cells to T1-T6 cells. T8 was identified as the only bona fide effector Th2 cell population (Th2 properties closeness), and T7 was identified as Treg cells with certain features shared by T8, such as high levels of ICOS and CTLA4 (Treg activity closeness). Notably, CD25 (IL2RA) was also shared by T7 and T8 (Figure 4, C-E), with a much higher level in T7, which is consistent with classical Tregs having high CD25 levels and activated $\mathrm{T}$ cells having intermediate levels. Focusing on markers of cellular trafficking (migration closeness), T8 had high levels of CXCR4 (15) but not CCR6 (16) or CCR8 (17). CCR6 was uniquely expressed by T7, and T7 and T8 coexpressed high levels of CCR4, suggesting the role of CCR4 in pathogenic T cell homing during allergic inflammation. Interestingly, T8 was specifically enriched by CD294/CRTH2/PTGDR2 (18) and a homing integrin $\alpha 4$ (ITGA4), implying the potential to block the migration and function of the T8 cells with CRTH2 antagonists (19) and anti-integrin $\alpha 4$ (natalizumab, ref. 20, or vedolizumab, ref. 21). Within T7 and T8, CD103 was almost never expressed by CD25 $5^{+}$cells (Supplemental Figure 7, A and B), suggesting that activated T7 and T8 cells are newly recruited to the inflamed tissue from the circulation (6).

T7 cells exhibited gene expression consistent with a memory Treg (22) signature of $\mathrm{CD}^{+}{ }^{+} \mathrm{FOXP}^{+} \mathrm{IL}^{-}-10^{+} \mathrm{CTLA} 4^{+} \mathrm{IL}-2 \mathrm{~A}$ $(\mathrm{CD} 25)^{+} \mathrm{IL}-1 \mathrm{R} 1 / \mathrm{R}^{+}$(Figure $\left.4, \mathrm{C}-\mathrm{E}\right)$, consistent with the overabundance of $\mathrm{CD}^{2} 5^{+} \mathrm{FOXP}^{+}$cells by flow cytometry (Figure 2, C-E). These cells unexpectedly expressed modest levels of TGFB1 (Supplemental Figure 8) despite high levels of IL1O (Supplemental Figure 12). Accordingly, T8 expressed IL1ORA and IL1ORB (Figure 4D), implying that T7 secretes IL-10 to suppress T8 and to help B cells class switch to IgG4 (23). Indeed, B cell class switching was one of the functional nodes exhibiting closeness with T7 (Supplemental Figure 9A). Importantly, at the single-cell level, the T8 gene expression profile suggested that T8 was largely $\mathrm{CD}^{+}$and predominantly GATA $3^{+} \mathrm{IL}-4^{+} \mathrm{IL}^{-} 5^{+} \mathrm{IL}-13^{+}$ $\mathrm{HPGDS}^{+} \mathrm{IL}-17 \mathrm{RB}^{+} \mathrm{CD} 40 \mathrm{LG}^{+} \mathrm{NFAT}^{+}{ }^{+} \mathrm{NF} \kappa 1^{+}{ }^{+} \mathrm{AHR}^{+} \mathrm{NR}^{2} \mathrm{C1}^{+} \mathrm{PPARG}^{+}$, uncovering the cellular source of the vast amounts of Th2-associated cytokines (Figure 3C). Notably, these sets of Th2 transcript signatures were completely absent in T1-T6 (Figure 4C and Supplemental Figure 4C), indicating that EoE was causally associated with a subset of overabundant tissue lymphocytes, identified as T7 (Treg) and T8 (effector Th2).
Transcriptome comparison of T7 and T8. Genome-wide expression profiles of $\mathrm{T} 7$ and $\mathrm{T} 8$ (RPKM $>2.0$ in any sample, FDRadjusted $P$ value $<0.05$ by Mann-Whitney, fold change $>16.0$, T8 vs. T7) identified 427 genes with differential expression between these 2 disease-specific T cell clusters; of these 427 differentially expressed genes, 395 were higher in T8 and 32 were higher in T7 (Supplemental Table 7). A volcano plot comparing the transcriptome of T7 and T8 illustrated 2 cohorts of critical genes specific to $\mathrm{T} 7$ and T8, with the more upregulated genes highlighting the superactivated state of T8 (Figure 4D). Besides the leading difference of transcription factors (FOXP3 versus GATA3), the differential genes between $\mathrm{T} 7$ and $\mathrm{T} 8$ were highlighted by genes that encoded for Th2 cytokines (e.g., IL4, IL5, IL13), inhibitory/activation markers (e.g., SLAMF1, CD4OLG), inhibitory cytokines (e.g., IL10), IL-1 receptor family members (e.g., IL1R1, IL1R2), acute activation proteins (e.g., JUN), classical Th2 markers (e.g., PTGDR2[CRTH2], IL17RB [IL25R], IL1RL1[ST2L], HPGDS), and the SCFA receptor FFAR3.

An unsupervised comparison of T7 and T8 versus all other clusters was performed with the goal of deciphering the biological functions of T7 and T8. Significant gene lists of T7 and T8 were filtered by fold change ( $>4.0, \mathrm{~T} 7+\mathrm{T} 8 \mathrm{vs}$. all others) followed by functional interrogation. Briefly, T7 was largely enriched in negative regulation of immune responses and cytokine biosynthesis, $\mathrm{T}$ cell selection, IgG isotype switching, regulation of $\mathrm{CD}^{+} \alpha \beta \mathrm{T}$ cell lineage commitment, $\mathrm{T}$ cell costimulation and activation, and positive regulation of IL-2 synthesis, with positive regulation of T cell immune responses also being identified (Supplemental Figure 9A). T8 was primarily enriched in Th2 cytokine production and exocytosis; $\mathrm{T}$ cell selection, activation, and differentiation; response to hypoxia; and a superactivated state due to inputs from NFкB, MAPK, and TLR pathways, as well as stimulation from lipid and calcium (Supplemental Figure 9B). The full functional nodes (screened by FDR-adjusted $P<0.05$, Fisher test-based) for defining T7 and T8 are presented in Supplemental Tables 8 and 9, respectively.

Classical transcription factor analysis of $T 7$ and T8. T8 expressed high levels of the key Th2 transcription factor GATA3, T7 expressed the highest levels of FOXP3, and T8 and T7 expressed equally high levels of MAF. Specifically, high levels of PPARG, AHR, NR3C1 (the bona fide glucocorticoid receptor), NFKBIZ, NFATC3, NFAT5, and $J U N$ were enriched in T8 (Figure 4, C-E), suggesting that T8 cells are acutely activated effectors that are particularly sensitive to lipids, including fatty acids, arachidonic acids, and aryl carbons (Supplemental Figure 9B). GATA3 was expressed at the highest levels in T8 but was also present in T7 cells. In contrast, FOXP3 was expressed in $\mathrm{T} 7$ and completely absent in T8 cells (Supplemental Figure 10, A-C). Expression of FOXP3 and GATA3 inversely correlated in T7 with the presence of the double-positive cells (Supplemental Figure 10, A and B). Notably, GATA3 ${ }^{+} \mathrm{FOXP} 3^{+} \mathrm{CD} 4^{+}$ $\mathrm{T}$ cells have been found in mice with impaired oral tolerance and enhanced allergic responses (24-26). The cumulative value (Sig$\mathrm{ma} / \Sigma$ ) for FOXP3 was highest in $\mathrm{T} 7$ and substantially lower in all other $\mathrm{T}$ cluster populations, whereas the cumulative value (Sig$\mathrm{ma} / \Sigma$ ) for GATA3 was highest in T8 but also present in all other populations (Supplemental Figure 10C). Flow cytometry demonstrated the presence and dichotomy of $\mathrm{FOXP3}^{+}$and $\mathrm{GATA}^{+}$events in EoE biopsy tissue confined to the CD4 compartment and the clear presence of double-positive cells (Supplemental Figure 10D). 


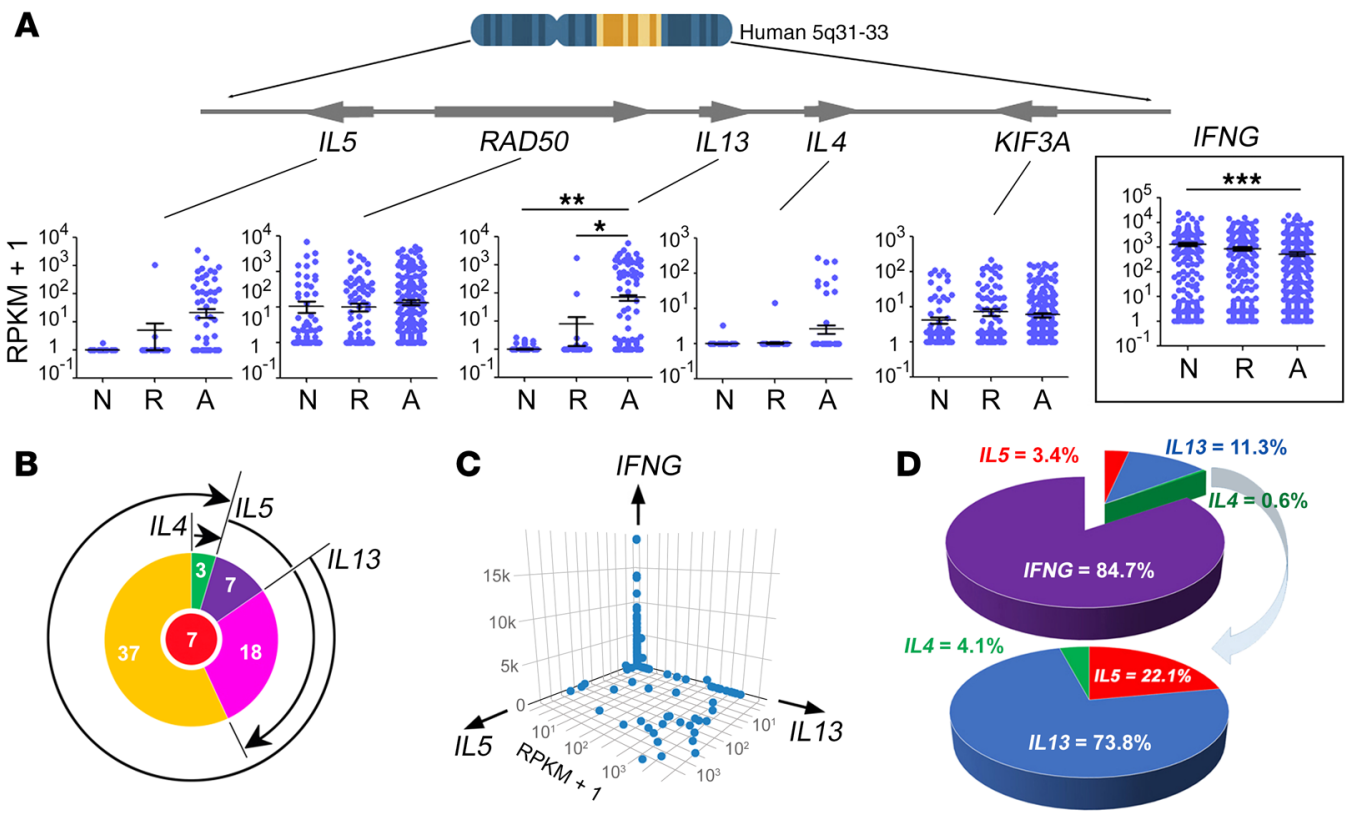

Figure 5. Single-cell Th2 cytokine mRNA analyses. To characterize the Th2 cytokine expression at mRNA and protein levels, relevant gene expression of 1088 tissue T cells at the single-cell level was analyzed along with independent FACS analyses. (A) Scrutinizing the human 5q31-33 Th2 cytokine cluster from IL5 to KIF3A, the RPKM scatter plots of all 5 adjacent genes in the context of disease activity (N, normal; R, remission; $A$, active EoE) are shown with each dot representing a single cell. The expression pattern for Th1 cytokine INFG (interferon $\gamma$ ) was plotted as a function of disease activity (mean $\pm \mathrm{SEM},{ }^{*} P<0.05,{ }^{*} P<0.01,{ }^{* *} P<0.001$; 1 -way ANOVA with Bonferroni's multiple comparison test). (B) Boolean logical analysis of Th2 cytokine-producing pattern of all Th2-capable cells ( $n=72$, center red circle represents 7 triple-cytokine-positive cells). (C) Three-way quantification of cytokine load by $\mathrm{CD}^{+} \mathrm{T}$ cells from allergic inflamed tissues encompassing the $1088 \mathrm{~T}$ cells. (D) Pie-chart break down of tissue T cell cytokine abundancy on the basis of single-cell RPKM enumeration.

T7 cells expressed markers not typically pertaining to Tregs, such as CD4OLG, IFNG, TNFA, and IL17A. These results at least partially support the idea that Tregs, by acquiring GATA3 expression during EoE allergic inflammation, may lose their immune-inhibitory properties and generate a transitional Th population that has an impaired potential of suppressive function and may exhibit characteristics of effector Th2 cells.

Th2 cytokine single-cell topology at mRNA levels. At both mRNA and protein levels, we aimed to uncover the Boolean pattern of Th2 cytokine expression by tissue $\mathrm{T}$ cells $(6,27)$, which are expected to be enriched within Th2 cells. We focused on human chromosome $5 \mathrm{q} 31-33$, as this region is genetically linked to susceptibility to allergic inflammation and is proximal to the Th2 cytokine cluster encoding IL-4, IL-5, and IL-13 (Figure 5A), which are highly relevant to allergic disease pathogenesis (28). The concentration of Th2 cytokine-capable (defined by detectable RPKMs on any Th2 cytokine loci) cells in EoE tissue reached a striking $12.3 \%$ of the $\mathrm{CD}^{+}$tissue lymphocytes (72/584, Figure 5A), corroborating the robust RPKM reads in the bulk RNA-seq analysis (Figure $3 \mathrm{C}$ ). Notably, this analysis resulted in a concentration of Th2 cells (defined by any Th2 cytokine RPKM detectable) approximately 2 times higher than the aforementioned $6.4 \%$ of $\mathrm{T} 8$ (defined as effector Th2 cells) by PCA in allergic tissue. Importantly, Th2 cytokine producers were barely detected in normal and remission tissue (Figure 5A), and other non-Th2 genes embedded in the Th2 cytokine loci, namely RAD5O and KIF3A, were not dysregulated in any disease con- texts (Figure 5A), indicating the specific control of gene expression in the $5 \mathrm{q} 31-33$ locus at the single-cell level. Despite encoding a Th1 cytokine, IFNG was abundantly expressed by tissue lymphocytes across all disease states (active and remission). IFNG was expressed primarily in T2-T5 cells, rarely in T6-T7 cells, and hardly ever in T8 cells (Supplemental Figure 8). Notably, occasional IFNG-expressing Tregs were present in $\mathrm{T} 7$ cells; this finding contrasted with the IFNG-negative phenotype in T8 effector Th2 cells (Supplemental Figure 8), suggesting a role of IFN- $\gamma$ in the Treg/T7 cell heterogeneity and instability previously shown (29).

Among the 584 EoE single cells randomly collected from 11 subjects with active EoE, there were 5.5\% IL-5-producing, $11.1 \%$ IL-13-producing, and $1.7 \%$ IL-4-producing Th2 cells (with overlapping spectrums, Figure 5, A-C). IL-5/IL-13 and IL-13/IL-4 double producers were $25 \%$ and $4 \%$ of all Th2 cytokine-producing capable cells isolated from tissue with active EoE, respectively. Beyond the double producers, we also identified $10 \%$ of the Th2 cytokine-producing capable cells as robust, triple-cytokine producers (Figure 5B). Human blood Th2 cells gain IL-4 capacity earlier than IL-5, which is deemed a terminal event during human Th2 differentiation (27). Relatedly, we did not detect the cellular presence of any single IL-4-producing cell, nor IL- $5^{+} \mathrm{IL}_{-} 4^{+}$double-positive cells. We explored the relationships of all single, double, and triple producers (Figure 5B). Nearly all cytokine-producing cells expressed IL-13, with approximately a quarter also expressing IL-5. Of note, no Th2 cytokines were expressed in the $\mathrm{T} 7$ population or in the $\mathrm{T} 1-$ T6 populations (Figure 4, C-E).

The $\mathrm{CD}^{+} \mathrm{T}$ cells doubled in concentration during active EoE compared with controls, and Th2 cytokine expression (IL4, IL5, and IL13) was primarily confined to the CD4 compartment (Supplemental Figure 11). There was complete dichotomy between IFNG and Th2 cytokine expression; few cells expressing IFNG expressed IL4, IL5, or IL13 (Figure 5C). IFNG was the most abundant cytokine produced by tissue lymphocytes, reaching $84.7 \%$ of total T helper cytokine reads (Figure 5D), followed by IL13 and IL5 (11.3\% and 3.4\%, respectively). IL 4 constituted less than $1 \%$ of total cytokine load, suggesting that $I L 4$ is critical only in the early induction phase of human Th2 inflammation. Interestingly, IL1O, which encodes a key negative regulatory cytokine, was expressed 
across T1-T8 at relatively moderate to high levels (Supplemental Figure 12A), with T7 having the highest and T8 having the lowest IL1O expression. The lack of IL1O in T8 is consistent with T8 being the Th2 cytokine source and IL-10 being a suppressive cytokine; indeed, IL1O expression was present in the other T clusters, primarily in $\mathrm{T} 7$ (Supplemental Figure 12A), and expression of $I L 10$ was mutually exclusive with that of Th2 cytokine genes at the single-cell level (Supplemental Figure 12E). Classical theory argues for the role of Tregs in synthesizing the antiinflammatory cytokines IL-10 and TGF- $\beta$, with the latter contributing to tissue remodeling. Surprisingly, single-cell mRNA data showed that both IL-10 and TGF- $\beta$ are not restricted to Tregs (T7 herein) in allergic tissue. Focusing on T7 (Treg) cells, IL1O was expressed at much higher levels than TGFB1 in the diseased tissue (Supplemental Figure 12, A-C). Moreover, no disease correlation was observed for IL1O and TGFB1, although FOXP3 is enriched in active disease (Supplemental Figure 12D), a finding that we corroborated at the protein level by FACS (Figure 2, C-E). IL-10 protein was also detected in a small branch of $\mathrm{CD}^{+} \mathrm{T}$ cells, although $\mathrm{CD} 8^{+} \mathrm{T}$ cells also produce it (Supplemental Figure 12B). The difference in observed abundancy in IL-10 levels (mRNA vs. protein) suggests that IL-10 may be regulated posttranscriptionally in vivo. Finally, TGFB1 and FOXP3 do not overlap with IL1O at the single-cell level, which was robustly produced by all $\mathrm{T}$ cell clusters more than expected (Supplemental Figure 12, A and D-F); thus, these data suggest that IL-10 and TGF- $\beta$ are differentially regulated at different levels in tissue Tregs.

Though mastocytosis typically accompanies allergic inflammation including EoE, there was barely any molecular evidence for Th9 cells, as indicated by the absence of IL9 expression in T1T8 (Supplemental Figure 8); likewise, no substantial IL6 and IL3 expression was observed in any T cell compartment. IL17A ${ }^{+}$cells were primarily confined to the CD8 populations, though a portion of $\mathrm{T} 7$ was also IL17A ${ }^{+}$. However, we did not observe any association with disease activity (data not shown). TGFB1 levels were detected to a moderate degree in $\mathrm{CD}^{+} \mathrm{T} 1-\mathrm{T} 6$, were the highest in T7, and were not detected in T8. IL2 was produced by T1-T7 at variable levels, whereas the IL-2 receptor (IL2RA/CD25) was primarily expressed by T7 and T8 (Figure 4, C-E and Supplemental Figure 8), consistent with FACS data that showed the increase of $\mathrm{CD} 4{ }^{+} \mathrm{CD} 25^{+}$events in active disease (Figure 2, C-E). Mild to moderate levels of IL17A were found across T1-T3 and T7 clusters but not in T4, T6, and T8 (Supplemental Figure 8).

Th2 cytokine single-cell topology at the protein level. A methodologic limitation of scRNA-seq is that it is focused on mRNA transcripts rather than protein levels. Therefore, we aimed to define Th2 cytokine production topology by tissue effector T cells using FACS. We first showed that the Th2 cytokine profiles are present at the protein level, thereby confirming 4 critical findings from our scRNA-seq experiments: (a) Th2 cytokine producers are dramatically enriched in allergic tissue compared with autologous blood (Figure 6A); (b) IL-13 is the dominant tissue cytokine compared with IL-4 and IL-5 (Figure 6B); (c) single T cells producing more than 1 Th2 cytokine are present (Figure 6B); and (d) the robust correlation of Th2 cytokines with disease activities is observed in the tissue, but not in the autologous blood $\mathrm{T}$ cell counterpart (Figure 6A).
We also aimed to elucidate the composition and developmental pathway of tissue $\mathrm{CD}^{+} \mathrm{T}$ cells in active EoE to identify the cytokine production framework. All active EoE flow cytometry.fcs files ( $n=13,>50,000 \mathrm{CD}^{+}$events collectively) were pooled for a SPADE (spanning-tree progression analysis of density-normalized events) analysis (30). A semiautomatic tree-building algorithm analyzed the developmental status of 5 parameters (CD8, IL-4, IL-5, IL-13, and IFN- $\gamma$ ), generating the tree based on the levels and relationship of the 5 leads without supervision. The first annotation resulted in a first-degree bifurcation of tissue lymphocyte 1 (TL1) and TL2 (Figure 6B), clearly and solely driven by the differential expression of CD4/CD8. The $\mathrm{CD}^{+} \mathrm{T}$ cell tree is shown with a heat diagram focused on 6 major cytokines of interest (Figure 6B). Focusing on in situ T effector differentiation, IL-4, IL-5, and IL-13 were produced by a small group of terminally differentiated $\mathrm{T}$ cells with largely overlapping but slightly different composition, among which IL-13 had the most robust production, potentially explaining its cellular and molecular dominance among Th2 cytokines (mRNA level: Figure 4, C-E, Figure 5, A-C, and Supplemental Figure 11; protein level: Figure 6). In the active disease tissue, all Th2 cytokines were produced by the TL1 arm with CD $4^{+}$identity (Figure 6B). Consistent with the scRNA-seq data (Figure 5A and Supplemental Figure 11), IFN- $\gamma$ was robustly produced and exhibited a complete dichotomy from Th2 cytokines. Both TL1/ $\mathrm{CD}^{+}$and $\mathrm{TL} 2 / \mathrm{CD}^{+}$are capable of producing IFN- $\gamma$ with $\mathrm{CD}^{+}$ cell molecular dominance (Figure 6B). IL-2, predominantly produced by $\mathrm{CD} 4^{+}$cells, was synthesized from a distinct branch of $\mathrm{TL} 1 / \mathrm{CD} 4^{+}$cells. These $\mathrm{CD} 4^{+}$cells transiently expressed IL-2 and were also capable of producing TNF- $\alpha$ and IFN- $\gamma$ at a later stage without any overlap with the Th2 branches. In contrast to Th2 cytokines, the differentiation topology of TNF- $\alpha$ and IFN- $\gamma$ is featured by robust coproduction by both CD 4 and CD8 with a CD8 dominance, explaining the abundancy of these 2 cytokines at the molecular and cellular levels (Figure 6B).

Assessing tissue TCR repertoires. We hypothesized that allergen-driven $\mathrm{T}$ cells would demonstrate some degree of TCR sharing and that this may be reflected in oligoclonality, especially as food allergens are primarily restricted to a limited group of approximately 8 allergenic food types. Unexpectedly, we found no evidence for this; instead, we found a trend for increased diversity among TCR clonal repertoires in patients with active EoE as shown by the increased Shannon entropy (Figure 7A, $P<0.05$ ). We found that patients with active disease had the most diversified TCR clonality, as their repertoires had the most clonotypes that occurred only once or twice $(n \leq 2)$ (Figure 7B). We examined TCR CDR3 length in order to gain insight into the maturation of $\mathrm{T}$ cell clonal responses across differentiation states (31). The CDR3 sequences of TCR- $\beta$ in patients with disease remission were significantly shorter than those of patients with active EoE and of normal controls $(P<0.05$, Figure $7 C)$. In contrast, the TCR $\beta$ CDR3 lengths from normal controls and patients with EoE were not significantly different from each other. It has been previously shown that TCR- $\beta$ CDR3 lengths reflected the T cell differentiation state in that longer CDR3 sequences were associated with a more naivelike phenotype and shorter CDR3 sequences were associated with a more effector memory-like phenotype (32). TCR- $\beta$ length negatively correlated with the degree of clonotype sharing among 
A

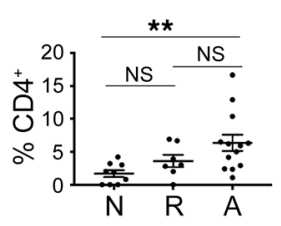

IL-5

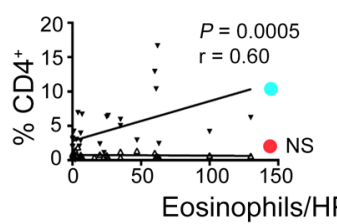

Eosinophils/HP

B
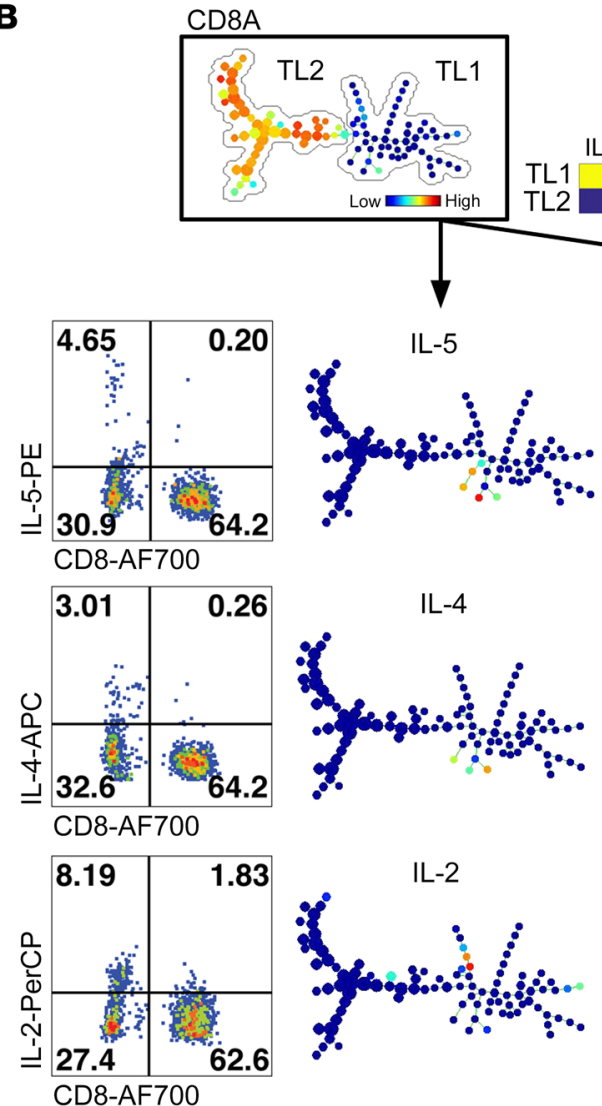

CD8-AF700

47.9

CD8-AF700

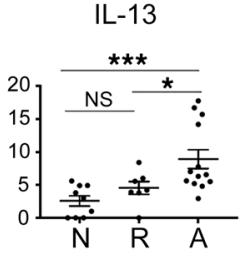

IL-13

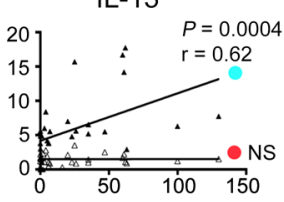

- Blood (autologous) - Tissue

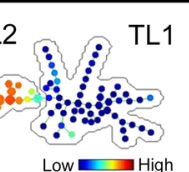

TL1
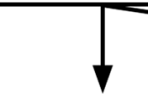

\begin{tabular}{l|lllll} 
& IL-4 IL-13 & CD8A IFN- $\gamma$ IL-5 High \\
\hline & & & & & \\
\hline & & & &
\end{tabular}
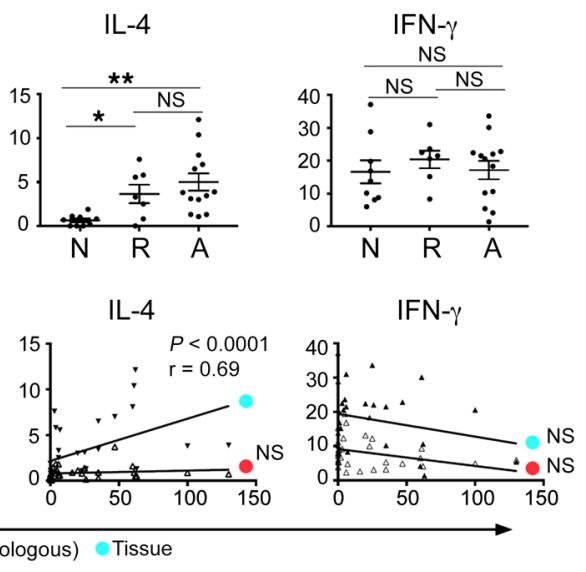

IL-5
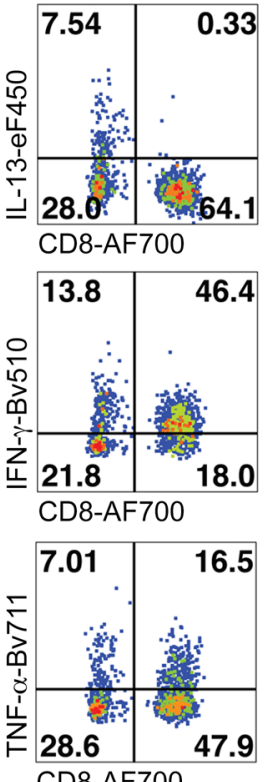

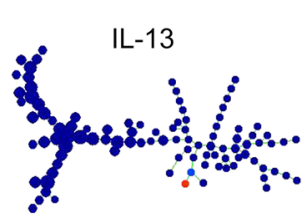

IFN- $\gamma$

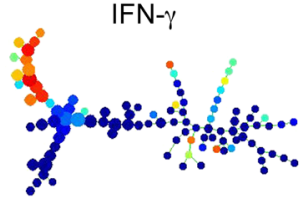

TNF- $\alpha$

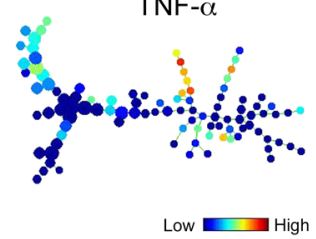

individuals (termed "publicity") and how close the TCR- $\beta$ was to the unmutated germline sequence. Thus, these data suggest that $\mathrm{T}$ cell clones from patients with disease remission were in a more differentiated state than those from patients with active disease and from normal controls. Moreover, we observed no sharing of TCR clonotypes among samples regardless of clinical designation. When classifying TCR clonotypes according to their T cluster designation, we found that though there was a degree of sharing of clonotypes among the T1-T6 clusters, there were no overlapping clonotypes between the T7 and T8 clusters nor between T7/T8 and the T1-T6 clusters (Figure 7D). The diversities (oligoclonality) of $\mathrm{T} 7$ and T8 were not different from T1-T6 despite being clonotypically distinct. These findings indicate that the $\mathrm{T} 7$ and $\mathrm{T} 8$ clusters are clonotypically distinct from T1-T6 and from each other, imply-
Figure 6. Single-cell Th2 cytokine protein analyses. (A) Top row: IL-4, IL-5, IL-13, and IFN- $\gamma$ protein was assessed by FACS in tissue $T$ cells from 29 subjects across 3 disease states. Major Th1/Th2 cytokine abundancy quantified (as \% CD4 $4^{+}$in the context of disease activities. N, normal; $\mathrm{R}$, remission; $\mathrm{A}$, active EoE (mean \pm SEM, ${ }^{*} P<0.05,{ }^{* *} P<0.01,{ }^{* * *} P<0.001$, 1-way ANOVA with Bonferroni's multiple comparison test). Bottom row: linear correlation between Th2 cytokine level and tissue eosinophilia, blood versus tissue. $P$ values and Spearman $r$ shown next to each correlation line. (B) All $\mathrm{CD}^{+}$ tissue lymphocytes from EoE were pooled for a SPADE (spanning-tree progression analysis of density-normalized events) analysis, identifying a dichotomy of tissue lymphocytes (TL) driven by CD8-CD4. The developmental trees for major EoE tissue cytokines were juxtaposed to representative FACS plots to reveal their levels, $T$ cell subtype source, and developmental patterns of each cytokine, with the heat color indicating expression levels and dot size indicating the cellular abundancy. ing that they may have unique allergen-specific properties mediated by their TCR interaction with their cognate food allergen.

We next examined TCR- $\beta$ V-J usage as a proxy for clonotype usage as a function of disease activity because no shared clones across disease states were found at the sequence level. This analytic approach extended beyond simply examining individual clonotype sequences because in the absence of a large number of sequenced $\mathrm{T}$ cells for each patient/sample, paired TCR- $\beta \mathrm{V}-\mathrm{J}$ usage may reflect overall clonotype selection without being constrained by exact sequence comparisons. PCA (Figure 7E) revealed that TCR- $\beta$ V-J usage by clonotypes in patients with remission was largely segregated from that of patients with active disease and control individuals, indicating that the remission $\mathrm{T}$ cells were fundamentally clonotypically different. In the same PCA, TCR- $\beta$ 

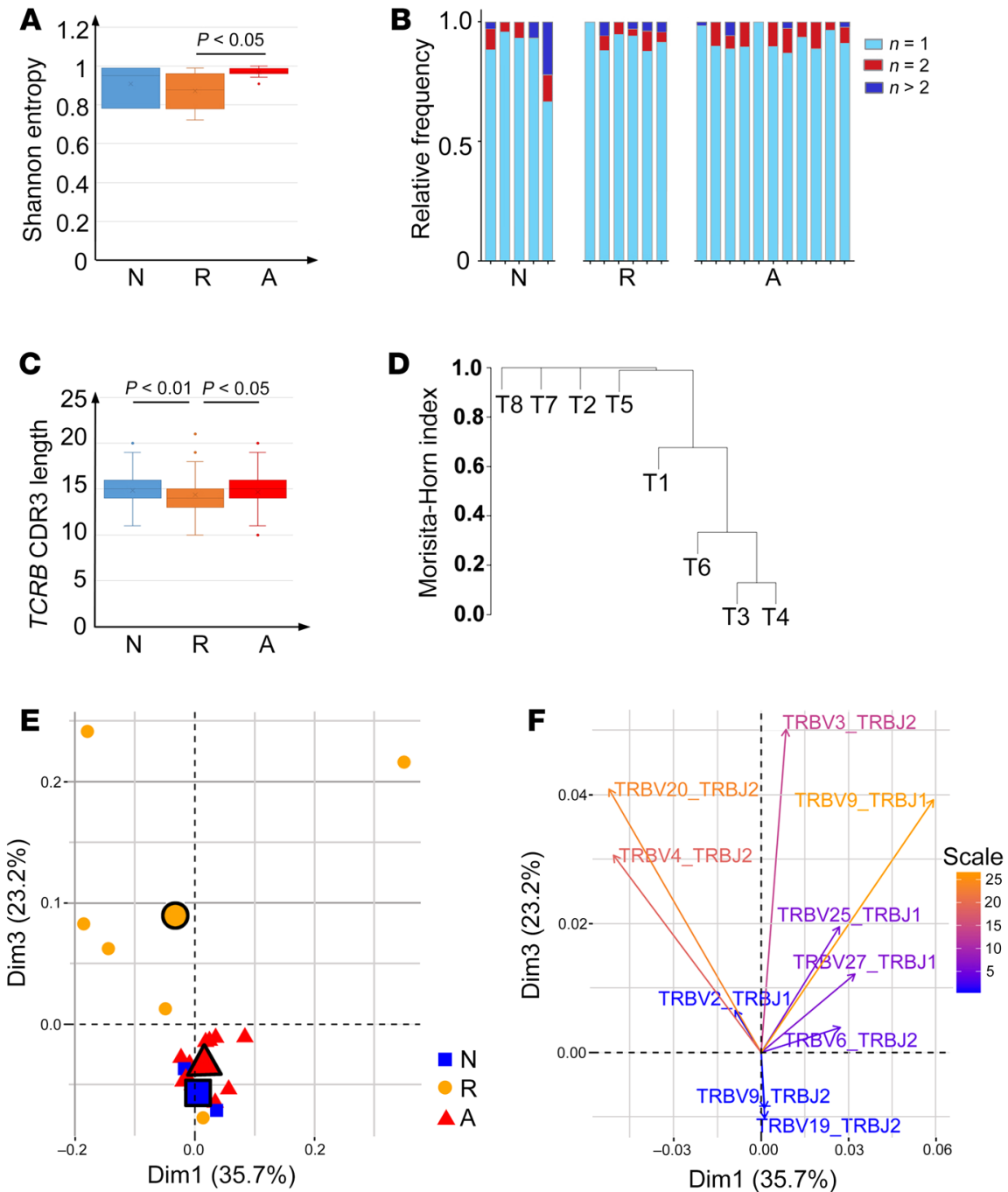

Figure 7. TCR clonotype analyses. (A) EoE TCR repertoire diversity (as represented by Shannon entropy normalized to the total number of cells) is shown as a function of disease activities (normal control [N], remission [R], active EoE [A]) (mean \pm SEM, 1-way ANOVA with Bonferroni's multiple comparison test). (B) Stacked bar charts representing stratified diversity for each TCR repertoire, in which the clonal occurrence of tissue TCR repertoire for all patients across 3 different disease activities were enumerated: unique ( $n=1$, cyan), duplicated ( $n=2$, red), and clonal ( $n>2$, blue). (C) The TCRB CDR3 lengths from sequence samples from NL, remission, and active EoE individuals were analyzed (statistical legend same as A). (D) The TCR repertoires of cells classified as T7 or T8 are distinct from T1T6 as measured by the Morisita-Horn dissimilarity index. Additionally, the T7 and T8 repertoires are distinct from each other in that they have no TCR clonal overlap. The Morisita-Horn dissimilarity index measures how dissimilar any 2 populations are based on overlapping species and the species' respective hierarchies. (E) Principal component analysis (PCA) of TRBV-TRB) usage shows that remission samples largely segregate from patients with active allergic inflammation and NL control samples based on TRBV-TRB] usage along dimension 1 (Dim1) and dimension 3 (black outlined shape, centroid). (F) On the basis of the PCA in E, a superimposed projection of all detected TRBV-TRBJ pairs indicating their disease state enrichment (remission sector: upper 2 quadrants; active and NL sectors: lower quadrants), shown by individual pair's Eigen vectors.
V4-J2, TCR- $\beta$ V20-J2, and TCR- $\beta$ V2-J1 pairs were enriched in the remission sector despite their distinct CDR3 sequences, whereas TCR- $\beta$ V9-J2 and TCR- $\beta$ V19-J2 pairs were enriched in the active disease and normal states (Figure 7F). V-J usage has been shown to be associated with the antigenicity of a given TCR (33). The uniqueness of the V-J usage during disease remission likely indicates recognition of different epitopes during this disease stage.

Taken together, single-cell TCR sequence analysis revealed that food allergen-driven $\mathrm{T}$ cells, at least in the context of this study, were likely driven by diverse antigenic stimulation. With respect to the greater diversity of TCR clonal repertoires in the active disease samples, a similar finding has been reported in TCR- $\beta$ repertoires in patients with celiac disease (34). Similarly, with normal regarded as the baseline diversity, the implication is that $\mathrm{T}$ cells in patients with active disease are potentially responding to a larger set of food-derived antigens than those in normal and remission individuals. A caveat, however, is that apparently higher diversity may arise from a lack of sampling depth, given that tissue-resident $\mathrm{CD} 3{ }^{+} \mathrm{CD} 45^{+} \mathrm{T}$ cells were much more abundant in the patients with active disease, but that the absolute number of cells sequenced remained largely unchanged. Our finding that the $\mathrm{T} 7$ and $\mathrm{T} 8$ clusters were clonotypically distinct reinforced the transcriptomic findings that they were 2 distinct subsets in terms of $\mathrm{T}$ cell differentiation and also suggested that a different set of epitopes was responsible for stimulating them. TCR- $\beta$ V-J usage was relatively independent of sequencing depth. Thus, the fact that the TCR- $\beta$ V-J usage differed between remission and active inflammation, as well as normal control, suggested that $\mathrm{T}$ cells in remission might recognize a different set of antigens than those in active inflammation and in normal control samples, and that the difference in the remission tissue may reflect an active role of TCR clonality in the process of disease remission and relapse.

Transcription factor topological screening identifies enrichment of lipid metabolism in type 2 cytokine-producing cells. We examined 1391 manually curated human transcription factors for their expression levels as a function of the T1-T8 categorization (Supplemental Figure 13A) (35). This analysis resulted in notably distinct transcription factor profiles within each single-cell $\mathrm{T}$ cluster, with only a small group of transcription factors common to all $\mathrm{CD}^{+} \mathrm{T}$ cell subtypes. Focusing on the active EoE-relevant T7 and T8, we identified T7- and T8-selective transcription factors (Supplemental Figure 13B). T8 was enriched in transcription factors involved in lipid metabolism and fatty acid response (Supplemental Figure 13C) based on expressions of NRF1, NCCR2, AHR, NCOA1, 


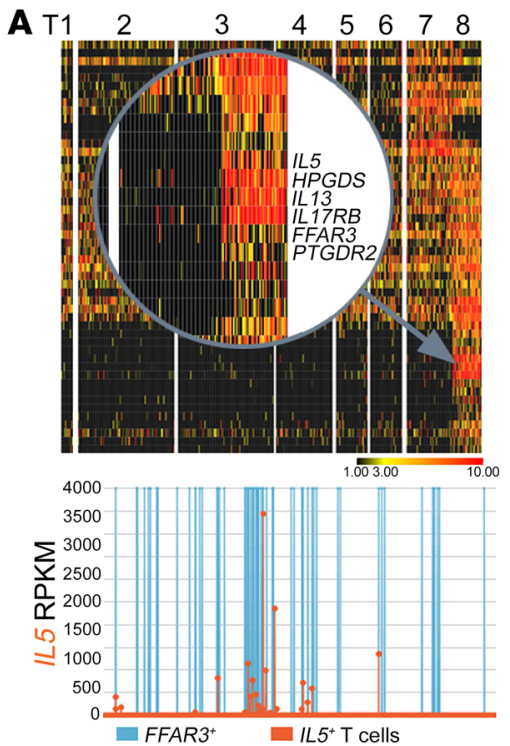

D

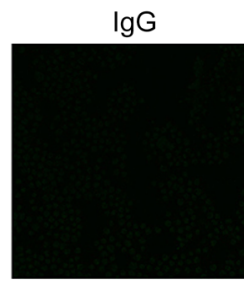

FFAR3

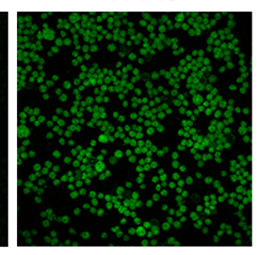

E
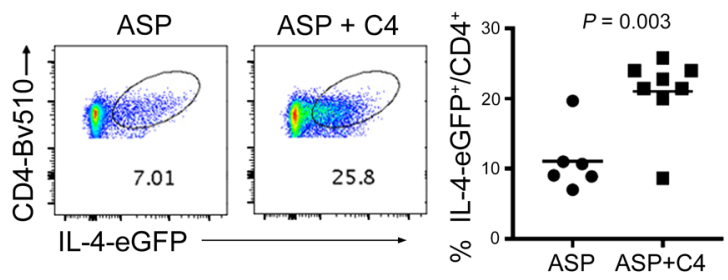

$\mathbf{F}$

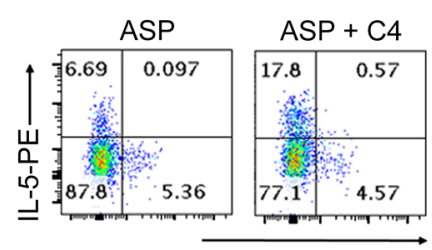

IFN- $\gamma-\mathrm{Bv} 711$
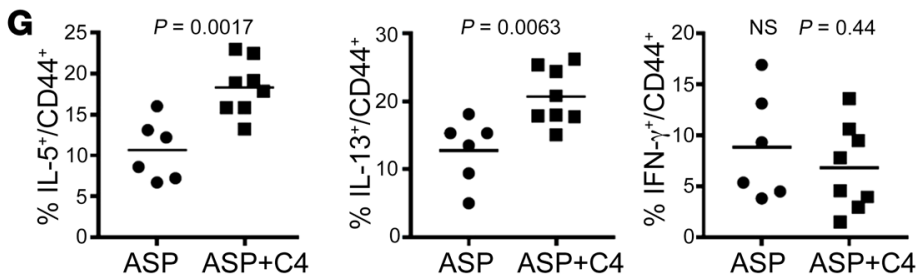

H

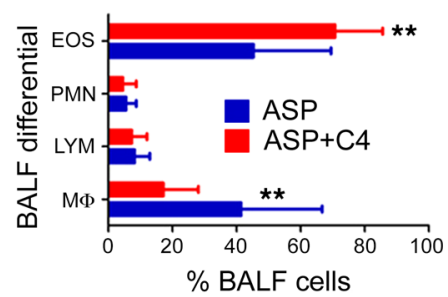

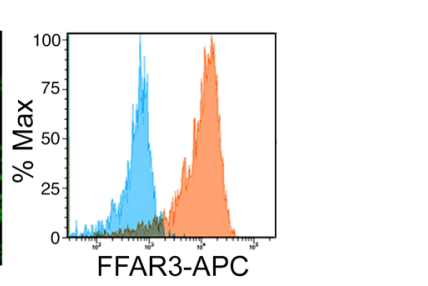
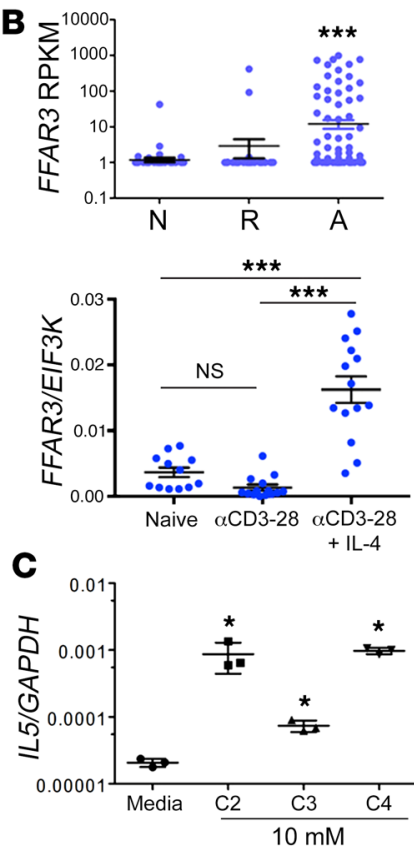

西

Figure 8. FFAR3 induction and Th2-enhancing effects of butyrate. (A) A magnified view in the upper panel emphasizing the IL5-correlating genes specifically present in the T8 cluster, with FFAR3 tracking with IL5 expression shown in the lower panel (orange, IL5 producers; blue, FFAR3 ${ }^{+}$T cells). (B) Upper panel: the single-cell expression patterns of $F F A R 3$ across 3 disease activity states ( $\mathrm{N}$, normal; R, remission; A, active EoE), with each data point representing 1 of the 1088 tissue T cells. Lower panel: with 3 days of Th2 differentiation (anti-CD3-CD28 [activated] with or without IL-4), human blood CD4 ${ }^{+} T$ cells isolated from patients with EoE upregulated FFAR3 transcript induced by $\alpha$ CD3-CD28 (activation) plus IL-4 but not $\alpha$ CD3-CD28 (activation) alone. (C) IL5 transcript production by human Jurkat cells following short-chain fatty acid exposures (C2, C3, and $\mathrm{C} 4$ each at $10 \mathrm{mM}$ for 24 hours). (D) Jurkat cells express FFAR3 on the cell surface by immunofluorescence and FACS. Original magnification, $\times 400$. (E) In a murine asthma model, eGFP-IL-4 reporter mice were aspergillus allergen-challenged (ASP-challenged) intranasally with and without C4 (1 mg coadministration). IL-4-eGFP expression in CD4+ cells was analyzed by FACS. (F and $\mathbf{G}$ ) Lung tissue CD4+ lymphocytes were assayed for Th1 and Th2 cytokine production by FACS. (H) The bronchoalveolar fluid (BALF) cells were quantified for major leukocyte populations. EOS, eosinophils; PMN, polymorphonuclear neutrophils; LYM, Iymphocytes; MФ, macrophages. All scatter plots are presented as mean \pm SEM, and all experiments were repeated at least 3 times $\left({ }^{*} P<0.05\right.$, ${ }^{* *} P<0.01,{ }^{* * *} P<0.001 ; 2$-tailed $t$ test).

NRIH4, RXRA, the glucocorticoid receptor [NRC31], 2 estrogen receptors [ESSRA and ESR1], and 3 members of the PPAR family [PPARG, PPARA, and PPARGC1A] (Supplemental Figure 13D). A Pearson correlation study of the expression patterns of the 1391 human transcription factors across $\mathrm{T} 1-\mathrm{T} 8$ indicated that $\mathrm{T} 1-\mathrm{T} 5$ are more related to each other and that T6, T7, and T8 differ from each other and from T1-T5 (Supplemental Figure 13E) (35).

Identification of the FFAR3/SCFA relationship with eosinophilia and $I L-5$. A cohort of 865 genes significantly correlated with levels of tissue eosinophils, a measure of EoE disease severity (Pearson correlation $P$ value $<0.05$, Supplemental Table 10; selected group shown in Supplemental Figure 10E). The gene cohort involves a classical Th2 marker PTGDR2 (CRTH2) ranked number $1\left(P=2.6 \times 10^{-5}\right)$ and a SCFA receptor FFAR3 ranked number $5(P=0.0002)$, followed by those genes encoding IL-25R (IL17RB), as well as HPGDS, IL-5, IL-13, IL-4, EGLN3, CFLAR-AS, LIF, SOCS1, TIGIT, CACNA1D, and CCL1. These data link eosinophilia with a series of mechanistically expected (e.g., CRTH2, HPGDS, SOCS1) and unexpected genes and pathways (e.g., EGLN3, CFLAR-AS, LIF, TIGIT, CACNAID, CCL1, and FFAR3). In light of these findings, the expression of FFAR3 robustly correlated with $I L 5$ at the single-cell level (Figure 8 A, Spearman $r=0.53, P<0.00001$ ).

Identification of a clinically relevant FFAR3/SCFA Th2-promoting pathway. FFAR3, conserved from rodents to humans, encodes a G protein-coupled receptor for SCFAs, with $\mathrm{C} 2-\mathrm{C} 5$ (named after the number of carbon atoms, ref. 36) being putative FFAR3 ligands. We first substratified all $\mathrm{T}$ cells within the context of EoE and found that FFAR3 is dysregulated in a pattern very similar to that of the Th2 cytokines (Figure 8B), with active disease exhibiting the high- 
est mean magnitude. We hypothesized that FFAR3 upregulation represents a positive regulator during Th2 cell differentiation. Intriguingly, we found that FFAR3 was induced by the key Th2 cytokine IL-4 (Figure 8B). We found that all SCFAs tested (C2C4) significantly increased the level of IL 5 transcripts produced by Jurkat cells compared with the level produced by media-treated controls (Figure 8C), with C4 having significantly higher efficacy than C3 ( $P<0.001,2$-tailed $t$ test). It is notable that $\mathrm{C} 2$ was able to induce IL-5, as C2, unlike C4, has little HDAC activity (15). Jurkat cells were naturally positive for FFAR3 protein (Figure 8D).

We aimed to substantiate the relationship between FFAR3SCFA by assessing the effect of $\mathrm{C} 4$ exposure in both human primary T cells as well as murine T cells, as the SCFA-FFAR3 axis is conserved in mice (37). For the murine system, splenocytes were CD4-purified and subsequently treated by 2 parallel paradigms to examine the effects of $\mathrm{C} 4$ during naive $\mathrm{T}$ cell $\mathrm{Th} 2$ differentiation and after Th2 differentiation (schematically depicted in Supplemental Figure 14A, top panel). Th2 cell differentiation was achieved by stimulating splenocytes with anti-CD3 and anti-CD28 in the presence of anti-IFN- $\gamma$ and IL-4; anti-IL- 4 was used as a negative control in some experiments. Subsequently, the major Th1/Th2 cytokines were measured by both intracellular FACS and quantitative RT-PCR. By FACS, intracellular IL-13 protein was robustly enhanced by $\mathrm{C} 4$ exposure during (Supplemental Figure 14A, paradigm I, left) and after Th2 induction (Supplemental Figure $14 \mathrm{~A}$, paradigm II, right), with strong trends for augmentation of IL- 4 and IL-5. Intriguingly, TNF- $\alpha$ was also robustly enhanced by $\mathrm{C} 4$ in both systems, corroborating its potential role as a Th2 inflammatory adjuvant. In contrast to Th2 cytokines, C4 had little enhancing effect on IFN- $\gamma$ and IL-2 production (Supplemental Figure 14A). Moreover, C4's enhancement on Th2 cytokines was dose dependent and IL-4 dependent, as replacing IL-4 with antiIL-4 during Th2 induction diminished the Th2-boosting effect of C4 (Supplemental Figure 14A). Following C4 stimulation after Th2 induction (paradigm II), IL-13 was also significantly boosted together with $\mathrm{TNF}-\alpha$, demonstrating a role of $\mathrm{C} 4$ on differentiated Th2 cells. In the same experiment, mRNA expression largely showed the same effect by $\mathrm{C} 4$; $\mathrm{C} 4$ specifically boosted Th2 cytokine expression levels (Supplemental Figure 14B), and this boost was partially abolished in the anti-IL-4 paradigm. Extending the system, peripheral blood-derived, CD4-purified human primary $\mathrm{T}$ cells were subjected to Th2 induction (as mentioned for murine $\mathrm{T}$ cells) in the presence and absence of C4. C4 induced IL-13 in a dose-dependent fashion as indicated by 2-way ANOVA analysis (Supplemental Figure 14C).

To examine whether the SCFA-induced Th2 augmentation was a conserved function in vivo, we induced allergic airway inflammation in IL-4 reporter mice (4get, ref. 38 ) in the absence and presence of $\mathrm{C} 4$. We found that mice cotreated with $\mathrm{C} 4$ had significantly higher intracellular IL-4 levels, an indicator for augmented Th2 characteristics, in the asthmatic compared with the control lung (Figure 8E). Moreover, by focusing on CD $44^{+}$-activated effector $\mathrm{T}$ cells isolated from the lung tissue and assessed by intracellular FACS, we demonstrated that $\mathrm{C} 4$ coadministration significantly increased IL- 5 and IL- 13 production by lung tissue $\mathrm{T}$ cells and evidenced a trend of decreased IFN- $\gamma$ production, in addition to boosting IL-4 production (Figure 8, F and G). Addi- tionally, C4 cotreated mice had significantly more eosinophilia and less macrophage infiltration in bronchoalveolar lavage fluid, indicating that a stronger Th2 polarization was induced by $\mathrm{C} 4$ cotreatment (Figure $8 \mathrm{H}$ ). These results collectively demonstrated a Th2-enhancing effect by the SCFA-FFAR3 axis in murine T cells during experimental allergic inflammation.

\section{Discussion}

Herein, we analyzed human tissue-residing $\mathrm{CD}^{+} \mathrm{T}$ cells using single-cell sequencing of isolated cells from the tissue of patients with active EoE compared with 2 control groups: patients in EoE remission and normal control individuals. We report a number of key findings, including (a) the presence of substantial $\mathrm{CD}^{+} \mathrm{T}$ cell heterogeneity, identifying 8 different cell populations (T1-T8 clusters); (b) identifying 2 populations of cells that were increased in patients with EoE (T7 and T8 clusters); (c) determining that these 2 populations of cells represented bona fide memory effector Th2 cells and a population of cells that resembled Tregs; (d) determining that these 2 populations of cells represented a substantial fraction of total $\mathrm{CD}^{+} \mathrm{T}$ cells in the tissue $(11 \%$ and $6 \%$ of tissue $\mathrm{CD}^{+} \mathrm{T}$ cells for $\mathrm{T} 7$ and $\mathrm{T} 8$, respectively), which was unexpectedly high given that the cells were isolated from patients without immediate allergen exposure at the time of procurement; (e) determining that a substantial percentage $(-30 \%)$ of $\mathrm{CD}^{+} \mathrm{T}$ effectors in active disease produced Th2 cytokines, which was remarkable given that prior studies had reported this level of cytokine expression only following multiple rounds of antigen stimulation in prolonged culture (27) and that tissue lymphocytes were directly subjected to RNA isolation right after isolation without being synchronized or stimulated ex vivo; (f) determining that the Treg-like cells and Th2 cells were transcriptionally related but distinguished by a series of TFs, with absolute dichotomy of FOXP3 expression; (g) elucidating the specific genes that distinguished these cells-Treg-like cells were characterized as $\mathrm{CD}^{+}$ $\mathrm{FOXP}^{+} \mathrm{IL}^{-10^{+}} \mathrm{CTLA}^{+} \mathrm{IL}-2 \mathrm{~A}(\mathrm{CD} 25)^{+} \mathrm{IL}-1 \mathrm{R} 1 / \mathrm{R}^{+}{ }^{+}$and Th2 cells as $\mathrm{CD}^{+} \mathrm{GATA}^{+}{ }^{+} \mathrm{IL}-4^{+} \mathrm{IL}^{-} 5^{+} \mathrm{IL}^{-} 13^{+} \mathrm{HPGDS}^{+} \mathrm{CRTH}^{+} \mathrm{IL}^{-17 \mathrm{RB}^{+} \mathrm{CD} 4 \mathrm{OLG}}$ $(\mathrm{CD} 154)^{+} \mathrm{NFAT1}^{+} \mathrm{NF}^{-}-\mathrm{B}^{+}{ }^{+} \mathrm{AHR}^{+} \mathrm{NR} 3 \mathrm{C}^{+}{ }^{+} \mathrm{PPARG}^{+}$by gene expression; (h) demonstrating that most $\mathrm{Th} 2$ cells robustly expressed IL-13, with the single-cell topology demonstrating IL-5 and IL-4 coexpression at mRNA and protein levels; (i) determining the properties of the TCR sequence at the single-cell level, providing evidence that EoE was not driven by a restricted class of TCR clones, at least in the context of the model disease (EoE) and the relatively low number of cells being examined; and finally (j) identifying evidence that effector Th2 cells were particularly sensitive and activated by a lipid signaling pathway, with a prominent role for SCFAs that can enhance Th2 cytokine production by tissue $\mathrm{T}$ cells likely via FFAR3.

One of the significant contributions of this study is the identification of $8 \mathrm{~T}$ cell subclasses, in particular, the disease-associated $\mathrm{CD} 4^{+}$populations (T7 and $\mathrm{T} 8$ ) whose concentrations increased in active disease. Our data indicated that EoE was largely a CD4 ${ }^{+}-$ driven disorder. Function wise, it is notable that T7 cells have properties of inhibitory cells but are apparently ineffective in the setting of this strong tissue allergic inflammatory response. Notably, these apparent Tregs express several unexpected markers such as $C D 154, I F N G$, and IL17A, suggesting that they may be 
antigen-experienced and contributory to the disease pathogenesis, rather than suppressive. It also remains possible that these Treg-like cells may be transitional toward T8 cells, consistent with recent proposals about Treg reprograming $(39,40)$. It is plausible to speculate that these Treg-like T7 cells may be initially recruited for negative immune regulation. Within the Th2 milieu, they might start the transition to Th2 cells, a linear transitional process initially demonstrated in the murine system (25). The strong local IgG4 response seen in EoE (41) may be mediated in part by these Treg-like cells via production of IgG4-promoting IL-10. It is notable that EoE also develops in the setting of oral immunotherapy for the treatment of food anaphylaxis (42), as Treg and IgG4 responses are also seen in this setting (23).

The importance of the FFAR3-SCFA axis in human allergic inflammation is an intriguing finding. Human intestinal epithelium, including the esophagus (43), is enriched with commensal microbiota, and SCFA (C2-C5) is a major metabolic product (44) of this microbiota. Recent data demonstrated that the esophagus is colonized by microbiota known to be butyrate producers (45), mainly comprising several genera of Clostridium under the Firmicutes phylum (46), especially Clostridium cluster IV and XIVa. Notably, C4-producing Firmicutes were 1 of the 4 main phyla in the esophagus of EoE and control patients (47). As a dietary supplement, C4 has been purposed as a protective antiinflammatory agent. IL-5 is the key pathogenic cytokine in allergic inflammation, and now humanized anti-IL-5 and anti-IL-5RA antibodies are approved for the treatment of severe asthma with an eosinophilic phenotype (48). Notably, FFAR3 was a top gene tracking with IL5 expression and tissue eosinophilia, and FFAR3 ligands exacerbated the severity of a murine asthma model. We propose that FFAR3 induction serves as a positive feedback loop in obtaining the full IL-5- and IL-13-producing capacity of differentiating effector Th2 cells in the tissue, resulting in a potentiated pro-Th2 inflammatory response following allergen exposure. The mechanistic pathway of IL- $4 \rightarrow$ FFAR3 $\rightarrow$ Th2 cytokines may be critical to enhance differentiation efficiency and serve as a Th2 amplifier when a robust Th2 response is needed. Our findings add to the growing literature linking SCFA with modulating the immune system (49) and are consistent with earlier work showing that butyrate induced IL-5 by altering histone acetylation in the $I L 5$ promoter of Jurkat cells (50). At the same time, butyrate has also been shown to inhibit Th2 cytokine production by type 2 innate lymphoid cells (ILC2s) (51) and to induce Treg inhibitory cell function via FFAR2 (52). This could be potentially explained by distinct roles of butyrate in different anatomical regions, as well as differences in the activity of butyrate when administered by different routes.

There are several limitations to the present study. First, single-cell transcriptome analysis may be influenced by the lack of synchronization of "firing timing/pattern" of each cell (53). Second, TRMs are known for their inaccessibility, with only a small portion of the cell $(<20 \%)$ being able to be recovered (54), which draws into question their representability. Third, this study was mainly focused on T7 and T8 due to their disease association, yet the other populations of $\mathrm{T}$ cells, T1-T6, deserve further scrutiny. Notably, the RPKM-based normalization method may underestimate the potency of the T8 effector Th2 cells, as they could potentially be more well-stocked than they appear on the heat diagram.
In addition, understanding the heterogeneity of the cells in the T7 and T8 clusters, such as the presence of Th2A cells (14), has not yet been undertaken. Limited by the biopsy size and availability, we were not able to perform functional suppression assays between $\mathrm{T} 7$ and $\mathrm{T} 8$, nor have we yet to use allergen tetramers to determine antigen specificity $(14,55)$. Finally, although the effect of butyrate is relatively specific for IL-5, its activity may be mediated at least in part by its ability to directly inhibit HDAC activity (15).

In conclusion, we have identified the involvement of 2 prominent populations of CD $4^{+} \mathrm{T}$ cells, Th2 effector cells and Treg-like cells, in the pathoetiology of EoE. We determined that Th2 cytokine production is largely confined to the $\mathrm{CD} 4^{+} \mathrm{Th} 2$ cell population, which represented approximately $30 \%$ of $\mathrm{CD} 4^{+}$cells, and that such cells expressed IL-13 robustly and IL-5 and IL-4 to a lesser extent. It is notable that this represents a large enrichment of Th2 cells compared with that of the peripheral blood. We are intrigued by the finding that Th2 cytokines were readily detectable without stimulation, as prior studies have found substantially lower levels and typically require in vitro stimulation to detect substantial cytokine production. We attribute the remarkable enrichment in cytokine production to the unique focus on tissue-residing cells. We are intrigued by the enrichment of the unique population of Treg-like cells, which appear to be a hallmark of EoE and ineffective at appropriately suppressing the adaptive response. We propose that this population may shift the balance from a classic type 2-associated, IL-4-mediated IgE allergic response to the chronic type 2 IL-5/IL-13-driven and IgG4-associated immune response seen in EoE. Finally, we generated evidence for a prominent role of lipid-induced cellular activation and signaling in Th2 cells and identified a role of SCFAs and their receptor FFAR3 in propagating Th2 cell-associated responses. Our findings challenge conventional wisdom that SCFAs are immunosuppressive. Since SCFAs are frequently encountered in the diet and are used in health-promoting food supplements, our findings deserve further attention. Though we are just beginning to dissect the significance of FFAR3 and the vast amount of single-cell data uncovered by our study, we hope that our reported findings will impact the understanding of the mechanisms modulating $\mathrm{T}$ cell fate in health and disease, and that further manipulating these cells will create better modalities to restore homeostasis.

\section{Methods}

See Supplemental Materials and Methods for additional information.

Study approval. Samples were obtained following informed consent, under the auspices of the IRB of the Cincinnati Children's Hospital Medical Center (CCHMC, 2016-0123). All human subject recruitments complied with relevant ethical regulations following our protocol approved by CCHMC's IRB. Written informed consent was received from participants prior to inclusion in the study.

Endoscopic biopsy processing. A single biopsy from the distal esophagus was collected into RPMI medium supplemented with $10 \%$ FBS, kept on ice, and transported to the research laboratory within 30 minutes for processing. The biopsy was then transferred into EDTA buffer for 15 minutes at $37^{\circ} \mathrm{C}$, washed once with PBS, minced, and then subjected to collagenase A digestion for 30 minutes. The resulting suspension was diluted with ice-cold PBS, passed through a 19-gauge needle, filtered through 2 layers of gauze, and washed with ice-cold PBS. 
Bioinformatic pipelines. All individual scRNA-seq raw FASTQ files (75 bp times 2, pair-ended) were aligned with the hg19 reference genome (UCSC). Transcript abundance was estimated using the Kallisto version (0.42.5) (56) against the UCSC (Gencode v26) transcriptome. Subsequent analyses were carried out by Strand NGS and Genespring GX 12. The large data sets (NGS) have been deposited to the Gene Expression Omnibus database with access number GSE126250.

\section{Author contributions}

MER, TW, and BJA conceived and designed the work. TW performed the sample processing, sorting, and single-cell capture experiments. TW and BJA analyzed the data and established the analytical strategy. PJD provided bioinformatic assistance supervised by BJA. YR, MR, and KK performed experiments. PP and VM contributed the blood and biopsy samples. HF and KR identified and gained written informed consent from participating patients. SD performed TCR-related analysis under the supervision of DD. MER and TW supervised the study.

\section{Acknowledgments}

We thank the Cincinnati Children's Hospital Medical Center's Gene Expression, DNA Sequencing and Genotyping, Research Flow Cytometry Core, and the Cincinnati Center for Eosinophilic Disorders. We thank Dana M. Dykes, Ajay Kaul, Michael K. Farrell, Tom Lin, Khalil El-Chammas, Maisam A. Abu-El-Haija, and Samuel A. Kocoshis for their clinical contributions. We thank Shawn Smith, Michael Eby, Tim Hubbel, and Shawna Hottinger for their assistance. This work was supported by the HOPE Research Award (APFED); NIH grants P30 DK078392, U19 AI070235, R01 AI124355, and R37 A1045898; the CURED Foundation; and the Sunshine Charitable Foundation and its supporters, Denise A. Bunning and David G. Bunning. We thank Fred Finkelman, Yui-Hsi Wang, Artem Barski, and Tal Shay for reviewing this manuscript.

Address correspondence to: Marc E. Rothenberg, Division of Allergy and Immunology, Cincinnati Children's Hospital Medical Center, 3333 Burnet Avenue, MLC 7028, Cincinnati, OH 45229. Phone: 513.636.7177; Email: rothenberg@cchmc.org
1. Ziegler SF. Division of labour by CD4(+) T helper cells. Nat Rev Immunol. 2016;16(7):403.

2. Furuta GT, Katzka DA. Eosinophilic esophagitis. N Engl J Med. 2015;373(17):1640-1648.

3. Hirano I, et al. Dupilumab efficacy and safety in adult patients with active eosinophilic esophagitis: a randomized double-blind placebo-controlled phase 2 trial. Presented at the American College of Gastroenterology annual scientific meeting. Orlando, Florida. October 16, 2017.

4. Rothenberg ME, et al. Intravenous anti-IL-13 mAb QAX576 for the treatment of eosinophilic esophagitis. J Allergy Clin Immunol. 2015;135(2):500-507.

5. Hirano I, et al. RPC4046, a monoclonal antibody against IL13, reduces histologic and endoscopic activity in patients with eosinophilic esophagitis. Gastroenterology. 2019;156(3):592-603.e10.

6. Mitson-Salazar A, et al. Hematopoietic prostaglandin D synthase defines a proeosinophilic pathogenic effector human $\mathrm{T}(\mathrm{H}) 2$ cell subpopulation with enhanced function. J Allergy Clin Immunol. 2016;137(3):907-918.e9.

7. Cheroutre H, Lambolez F, Mucida D. The light and dark sides of intestinal intraepithelial lymphocytes. Nat Rev Immunol. 2011;11(7):445-456.

8. Schön MP, et al. Mucosal T lymphocyte numbers are selectively reduced in integrin alpha E (CD103)-deficient mice. J Immunol. 1999;162(11):6641-6649.

9. Panina-Bordignon $\mathrm{P}$, et al. The $\mathrm{C}-\mathrm{C}$ chemokine receptors CCR4 and CCR8 identify airway $\mathrm{T}$ cells of allergen-challenged atopic asthmatics. J Clin Invest. 2001;107(11):1357-1364.

10. Jagodziński PP, Trzeciak WH. Differential expression of chemokine receptor CXCR 4 in Th1 and Th2 subtypes of CD4+ lymphocytes. Folia Histochem Cytobiol. 2000;38(1):21-23.

11. Wang YH, et al. A novel subset of CD4(+) T(H)2 memory/effector cells that produce inflammatory IL-17 cytokine and promote the exacerbation of chronic allergic asthma. JExp Med. 2010;207(11):2479-2491.
12. Overgaard NH, Jung JW, Steptoe RJ, Wells JW. CD4+/CD8+ double-positive T cells: more than just a developmental stage? J Leukoc Biol. 2015;97(1):31-38.

13. Jamieson AR, Giger ML, Drukker K, Li H, Yuan Y, Bhooshan N. Exploring nonlinear feature space dimension reduction and data representation in breast Cadx with Laplacian eigenmaps and t-SNE. Med Phys. 2010;37(1):339-351.

14. Wambre E, et al. A phenotypically and functionally distinct human $\mathrm{TH} 2$ cell subpopulation is associated with allergic disorders. Sci Transl Med. 2017;9(401):eaam9171.

15. Nakase H, Mikami S, Chiba T. Alteration of CXCR4 expression and Th1/Th2 balance of peripheral CD4-positive T cells can be a biomarker for leukocytapheresis therapy for patients with refractory ulcerative colitis. Inflamm Bowel Dis. 2009;15(7):963-964.

16. Chung SH, Chang SY, Lee HJ, Choi SH. The $\mathrm{C}-\mathrm{C}$ chemokine receptor 6 (CCR6) is crucial for Th2-driven allergic conjunctivitis. Clin Immunol. 2015;161(2):110-119.

17. Zingoni A, et al. The chemokine receptor CCR8 is preferentially expressed in Th2 but not Th1 cells. J Immunol. 1998;161(2):547-551.

18. Cosmi L, Annunziato F, Galli MIG, Maggi RME, Nagata K, Romagnani S. CRTH2 is the most reliable marker for the detection of circulating human type $2 \mathrm{Th}$ and type $2 \mathrm{~T}$ cytotoxic cells in health and disease. Eur J Immunol. 2000;30(10):2972-2979.

19. Kupczyk M, Kuna P. Targeting the PGD2/ CRTH2/DP1 signaling pathway in asthma and allergic disease: current status and future perspectives. Drugs. 2017;77(12):1281-1294.

20. Chen Y, et al. Asymptomatic reactivation of JC virus in patients treated with natalizumab. $N$ Engl JMed. 2009;361(11):1067-1074.

21. Singh H, Grewal N, Arora E, Kumar H, Kakkar AK. Vedolizumab: a novel anti-integrin drug for treatment of inflammatory bowel disease. J Nat Sci Biol Med. 2016;7(1):4-9.
22. Boix-Giner F, et al. High frequency of central memory regulatory $\mathrm{T}$ cells allows detection of liver recipients at risk of early acute rejection within the first month after transplantation. Int Immunol. 2016;28(2):55-64.

23. Wright BL, et al. Food-specific IgG4 is associated with eosinophilic esophagitis. J Allergy Clin Immunol. 2016;138(4):1190-1192.e3.

24. Krishnamoorthy N, et al. Early infection with respiratory syncytial virus impairs regulatory $\mathrm{T}$ cell function and increases susceptibility to allergic asthma. Nat Med. 2012;18(10):1525-1530.

25 . Noval Rivas M, et al. Regulatory T cell reprogramming toward a Th2-cell-like lineage impairs oral tolerance and promotes food allergy. Immunity. 2015;42(3):512-523.

26. Pelly VS, et al. Interleukin 4 promotes the development of ex-Foxp3 Th2 cells during immunity to intestinal helminths. JExp Med. 2017;214(6):1809-1826.

27. Upadhyaya B, Yin Y, Hill BJ, Douek DC, Prussin C. Hierarchical IL-5 expression defines a subpopulation of highly differentiated human Th2 cells. J Immunol. 2011;187(6):3111-3120.

28. Wills-Karp M, et al. Interleukin-13: central mediator of allergic asthma. Science. 1998;282(5397):2258-2261.

29. Daniel V, Wang H, Sadeghi M, Opelz G. Interferon-gamma producing regulatory $\mathrm{T}$ cells as a diagnostic and therapeutic tool in organ transplantation. Int Rev Immunol. 2014;33(3):195-211.

30. Qiu P, et al. Extracting a cellular hierarchy from high-dimensional cytometry data with SPADE. Nat Biotechnol. 2011;29(10):886-891.

31. Reynolds C, et al. Elongated TCR alpha chain CDR3 favors an altered CD 4 cytokine profile. BMC Biol. 2014;12:32.

32. Afik S, et al. Targeted reconstruction of $\mathrm{T}$ cell receptor sequence from single cell RNA-seq links CDR3 length to T cell differentiation state. Nucleic Acids Res. 2017;45(16):e148.

33. Han Y, et al. Identification of characteristic TRB $\mathrm{V}$ usage in HBV-associated HCC by using differ- 
ential expression profiling analysis. Oncoimmunology. 2015;4(8):e1021537.

34. Yohannes DA, et al. Deep sequencing of blood and gut $\mathrm{T}$-cell receptor $\beta$-chains reveals gluten-induced immune signatures in celiac disease. Sci Rep. 2017;7(1):17977.

35. Vaquerizas JM, Kummerfeld SK, Teichmann SA, Luscombe NM. A census of human transcription factors: function, expression and evolution. Nat Rev Genet. 2009;10(4):252-263.

36. Scheppach W. Effects of short chain fatty acids on gut morphology and function. Gut. 1994;35(1 Suppl):S35-S38.

37. Ang Z, et al. Human and mouse monocytes display distinct signalling and cytokine profiles upon stimulation with FFAR2/FFAR3 shortchain fatty acid receptor agonists. Sci Rep. 2016;6:34145.

38. Croxford AL, Buch T. Cytokine reporter mice in immunological research: perspectives and lessons learned. Immunology. 2011;132(1):1-8.

39. Barbi J, Pardoll D, Pan F. Treg functional stability and its responsiveness to the microenvironment. Immunol Rev. 2014;259(1):115-139.

40. Noval Rivas M, Chatila TA. Regulatory T cells in allergic diseases. J Allergy Clin Immunol. 2016;138(3):639-652.

41. Rosenberg CE, et al. Esophageal IgG4 levels correlate with histopathologic and transcriptomic features in eosinophilic esophagitis. Allergy. 2018;73(9):1892-1901.

42. Burk CM, et al. Eosinophilic esophagitis during peanut oral immunotherapy with omalizumab. J Allergy Clin Immunol Pract. 2017;5(2):498-501.

43. May M, Abrams JA. Emerging insights into the esophageal microbiome. Curr Treat Options Gastroenterol. 2018;16(1):72-85.

44. Ríos-Covián D, Ruas-Madiedo P, Margolles A, Gueimonde M, de Los Reyes-Gavilán CG, Salazar N. Intestinal short chain fatty acids and their link with diet and human health. Front Microbiol. 2016;7:185.

45. Nobel YR, et al. Increasing dietary fiber intake is associated with a distinct esophageal microbiome. Clin Transl Gastroenterol. 2018;9(10):199.

46. Van den Abbeele P, et al. Butyrate-producing Clostridium cluster XIVa species specifically colonize mucins in an in vitro gut model. ISME J. 2013;7(5):949-961.

47. Harris JK, et al. Esophageal microbiome in eosinophilic esophagitis. PLoS One. 2015;10(5):e0128346.

48. Rothenberg ME. Humanized anti-IL-5 antibody therapy. Cell. 2016;165(3):509.

49. Corrêa-Oliveira R, Fachi JL, Vieira A, Sato FT, Vinolo MA. Regulation of immune cell function by short-chain fatty acids. Clin Transl Immunology. 2016;5(4):e73.

50. Han S, et al. HDAC inhibitors TSA and sodium butyrate enhanced the human IL-5 expression by altering histone acetylation status at its promoter region. Immunol Lett. 2007;108(2):143-150.

51. Thio CL, Chi PY, Lai AC, Chang YJ. Regulation of type 2 innate lymphoid cell-dependent airway hyperreactivity by butyrate. J Allergy Clin Immunol. 2018;142(6):1867-1883.e12.

52. Arpaia N, et al. Metabolites produced by commensal bacteria promote peripheral regulatory T-cell generation. Nature. 2013;504(7480):451-455.

53. Vallejos CA, Risso D, Scialdone A, Dudoit S, Marioni JC. Normalizing single-cell RNA sequencing data: challenges and opportunities. Nat Methods. 2017;14(6):565-571.

54. Schenkel JM, Masopust D. Tissue-resident memory T cells. Immunity. 2014;41(6):886-897.

55. Wambre E, DeLong JH, James EA, LaFond RE, Robinson D, Kwok WW. Differentiation stage determines pathologic and protective allergen-specific CD4+ T-cell outcomes during specific immunotherapy. J Allergy Clin Immunol. 2012;129(2):544-551.e1.

56. Bray NL, Pimentel H, Melsted P, Pachter L. Near-optimal probabilistic RNA-seq quantification. Nat Biotechnol. 2016;34(5):525-527. 